\title{
Die multimodale Konstitution eines imaginären Raums als interaktive Problemlösung
}

\begin{abstract}
In linguistischen Untersuchungen erscheint sprachliche Raumreferenz gemeinhin als eine Aktivität, die notwendig ist, um dem Hörer/Leser die räumliche Lokalisierung von Objekten zu ermöglichen. In diesem Beitrag wollen wir zeigen, dass die sprachliche und kinesische Konstitution von Raum in der multimodalen Interaktion eine flexible kommunikative Ressource ist, die auch unabhängig von solchen Referenz-identifizierenden Erfordernissen eingesetzt werden kann.

Anhand der Videoaufnahme einer Lehr-Lern-Interaktion zeigen wir, wie die sprachliche und körperlich enaktierende Konstitution eines imaginären Raums eingesetzt wird, um eine komplexe Sachverhaltsdarstellung auf einen spezifischen Adressaten und dessen Verständnisprobleme zuzuschneiden. Im Zentrum der Datenanalyse steht zum einen die Rekonstruktion der interaktionsstrukturellen Einbettung der Raumkonstitution, die verdeutlicht, dass sie als Verfahren kommunikativ verwendet wird, weil andere Verfahren der Vermittlung abstrakter Sachverhalte bereits gescheitert sind. Zum anderen zeigen wir, wie sprachliche und kinesische Aktivitäten der Akteure systematisch bei der multimodalen Raumkonstitution zusammenwirken und wie durch sie vier unterschiedliche Dimensionen von Raum in der Interaktion (objektiv-physikalischer Raum, Interaktionsraum, individueller Verhaltensraum und imaginärer Raum) organisiert und aufeinander bezogen werden.

Dabei wird deutlich, dass die sozialsymbolische Kodierung von Räumen und die Überblendung verschiedener Dimensionen von „Raum“ als rekurrente Phänomene in der multimodalen Raumkonstitution eingesetzt werden.
\end{abstract}

\section{Einleitung}

In linguistischen Untersuchungen erscheint sprachliche Raumreferenz gemeinhin als eine Aktivität, die notwendig ist, um dem Hörer/Leser die räumliche Lokalisierung von Objekten zu ermöglichen und diese zu identifizieren (vgl. z.B. Linde/Labov 1975; Herrmann/Schweizer 1998; Levinson 2003; Fricke 2007; Berthele 2007; Tenbrink 2007, S. 183-199). Forschungsparadigmen sind die Untersuchung von per Instruktion elizitierten Wegauskünften, Raumbeschreibungen oder Objektlokalisierungen. Der Verweis auf Räumliches und seine Beschreibung sind in solchen Kontexten die primäre und meist auch die alleinige Interaktionsaufgabe. Die bisherige Forschung zur sprachlichen Raumkonstitution erweckt daher den Eindruck, als werde Raumreferenz stets um ihrer selbst willen unternommen. ${ }^{1}$ Folglich

$1 \quad$ Anders ist es im Falle der Erforschung räumlicher Metaphorik (vgl. z.B. Lakoff/Johnson 1980; Tenbrink 2007). Dort stellt sich natürlich die Frage, welche spezifische Funktionen der 
entsteht in derartigen Untersuchungen gar nicht erst die Frage, welche Funktion der Raumkonstitution interaktiv zukommt, ob es nicht auch Fälle von Raumkonstitution gibt, die nicht durch primär raumbezogene Informationsbedürfnisse motiviert sind, und wie sich solche andersartigen Motivationen in der Art und Weise der Raumkonstitution reflektieren.

In diesem Beitrag befassen wir uns mit einer solchen andersartigen Motivation von Raumkonstitution in der Interaktion. Wir möchten zeigen, dass die sprachliche und leibliche Konstitution von Raum in der multimodalen Interaktion eine flexible kommunikative Ressource ist, die auch unabhängig von Erfordernissen der Lokalisierung und Identifikation von Referenten zur Vermittlung komplexer abstrakter Konzepte eingesetzt werden kann. Dabei wird deutlich, dass die Funktion der Raumkonstitution und ihre konkrete Ausgestaltung nur aufgrund ihrer Einbettung in eine Interaktionssequenz zu verstehen ist. Die Raumkonstitution ist damit das Produkt einer Interaktionsgeschichte, auf die sie in funktionaler wie reflexiver Weise reagiert.

Grundlage unserer Untersuchung ist die Videoaufnahme einer LehrLern-Interaktion, in der vom Dozenten ein imaginärer Raum schrittweise konstituiert wird. Wir stellen zunächst die interaktive Vorgeschichte der Raumkonstitution im Fallbeispiel dar (Abschnitt 2). Sie zeigt, dass Raumkonstitution hier kommunikativ verwendet wird, nachdem andere Verfahren der Vermittlung abstrakter Sachverhalte gescheitert sind. Anschließend folgt die detaillierte Analyse der einzelnen Schritte der multimodalen Konstitution eines imaginären Raums (Abschnitt 3-4). Deren Abschluss bildet die Reformulierung der räumlichen Konstitutionsspezifik als Ergebnis der Bearbeitung basaler Anforderung der Etablierung und Ausgestaltung eines imaginären Raumes unter den faktischen Bedingungen von Interaktion (Abschnitt 5). Wir abstrahieren dann, wie sprachliche und kinesische Aktivitäten der Akteure systematisch bei der multimodalen Raumkonstitution zusammenwirken und wie durch sie vier unterschiedliche Dimensionen von Raum in der Interaktion (Umgebungsraum, Interaktionsraum, individueller Verhaltensraum und imaginärer Raum) organisiert und aufeinander bezogen werden (Abschnitt 6-7). Schließlich weisen wir die interaktive Funktion der Raumkonstitution als Verfahren eines dozentenseitigen recipient design aus und diskutieren, wieso sie als interaktives Verfahren der Vermittlung komplexer, abstrakter Konzepte besonders geeignet ist (Abschnitt 8).

Verwendung von ,Raum‘ als konzeptueller Ressource für die Repräsentation der Verhältnisse in einer Zieldomäne leistet. Unsere Untersuchung bezieht sich jedoch nicht auf räumliche Metaphorik, sondern auf die Konstruktion imaginärer Räume. 


\section{Die vorausgehende Interaktionsgeschichte: Gescheiterte Versuche einer Konzeptvermittlung}

Wir analysieren einen Ausschnitt aus einer Arbeitssitzung von vier Filmstudenten und zwei Dozenten (Regie, Drehbuch) in einer Filmhochschule. Im Rahmen dieser so genannten „Pitchings“ (Heidtmann 2009) werden Ideen für Filme entwickelt. Im Verlaufe eines solchen Pitchings entsteht ein interaktives Problem: Einer der Dozenten (DO) bemerkt, dass der Drehbuchautor (ST) den Ausdruck „Struktur“ im alltagsweltlich-indifferenten Sinne benutzt, nicht aber entsprechend seiner fachlichen Terminologisierung einer dramaturgischen Struktur mit einem Helden, einem Ziel, einem Hindernis und Problemfaktoren. Der Dozent versucht daraufhin, den Studenten auf unterschiedliche Weise auf die problematische Verwendungsweise aufmerksam zu machen und ihm die Relevanz von Struktur im dramaturgischen Sinne zu vermitteln. ${ }^{2}$ Als seine Vermittlungsbemühungen scheitern, ändert der Dozent seine Strategie. Er liefert mit dem Beginn von „Romeo und Julia“ (nach der Version „William Shakespeare's Romeo and Juliet“ (Regie: Baz Luhrman) mit Leonardo DiCaprio in der Rolle des Romeo aus dem Jahre 1996) ein Beispiel für eine gelungene Exposition, das er als narrative Performance gestaltet. Dabei konstituiert er schrittweise einen komplexen imaginären Raum, in dem er unterschiedliche Charaktere des Dramas zueinander positioniert und agieren lässt.

\subsection{Die interaktive Vorgeschichte: problematischer Strukturbegriff}

\subsubsection{Der Anfangsverdacht: Distanzierung von der Ausdrucksverwendung}

Der Drehbuchautor hatte eine Geschichte vorgelegt, die von den Dozenten harsch kritisiert worden war. Gegen die Kritik der Dozenten führte er den Begriff der „Struktur“ ins Feld: für mich das interessanteste an dem was wir da abgegeben haben is die struktur. Diese Verwendung führt beim Dozenten offenbar zum Anfangsverdacht, der Drehbuchautor verstehe den Strukturbegriff nicht im dramaturgischen Sinne und wisse insbesondere nicht, was er für die Konstruktion seiner Geschichte impliziere. Diesen Verdacht expliziert er jedoch nicht; vielmehr gibt er zu verstehen, dass er die mit dem Strukturbegriff implizierte Anforderung an eine Geschichte im vorliegenden Drehbuch nicht bewältigt sieht.

\footnotetext{
$2 \quad$ Ausschnitte dieses Beispiels werden als dozentenseitiges Verfahren der Wissensvermittlung
} in Heidtmann (2009, S. 218-220) beschrieben. 


\subsubsection{Stabilisierung einer Partnerhypothese: Nachfrage}

Der Drehbuchautor insistiert wenig später wiederum auf der Qualität des Drehbuchs unter Verweis auf dessen struktur. Der Dozent formuliert daraufhin seine Zweifel am Strukturverständnis des Studenten ich bin nicht SICHer ob sie- (.) das wort strukTUR wirklich: inhaltlich MEInen; und fragt dann explizit nach wenn sie jetzt strukTUR, meinen sie das im sinne von dramaTURgischer strukTUR? Die lapidare Antwort des Drehbuchautors ( $j a$ ja) scheint dem Dozenten zu bestätigen, dass der Student kein adäquates Verständnis von „Struktur" hat.

\subsubsection{Strategiewechsel I: Maieutisches Fragen und Kritik}

Der Dozent geht nun zu einem maieutischen Verfahren über (vgl. Hanke 1991). Hatte er bis dato versucht, den Studenten durch die Formulierung seiner Zweifel zur Selbsterkenntnis zu bringen, thematisiert er nun nicht mehr den Strukturbegriff als solchen. Vielmehr stellt er jetzt Fragen nach der Exposition der Geschichte, die darauf abzielen, dass der Student die Unangemessenheit ihrer Gestaltung selbst erkenne. Die maieutische Strategie führt jedoch auch nicht dazu, dass dieser den strukturellen Aufbau seines Drehbuchs als problematisch erkennt. Der Dozent formuliert schließlich eine deutliche Kritik an der Struktur der Exposition $<<t, k$ narrend $>a ̈ b$ der der der der $>$ MÜLLtransporter, (---) erZÄHLT uns übe:r; (-) unseren HAUPTcharakter nix. (Z. 097-100, Anhang). ${ }^{3}$ Diese wird vom Drehbuchautor jedoch bagatellisierend zurückgewiesen der DER ä:b- (-) legt en bisschen <<all>ne STIMM > $\downarrow$ ung an; (-) einfach- (Z. 103-106).

\subsubsection{Strategiewechsel II: Beispielnarration}

Der Dozent markiert daraufhin, dass stimmung keine Kategorie sei, die für die dramatische Struktur relevant ist. Er wechselt erneut seine Vermittlungsstrategie. Er geht nun zu einem Beispiel, der Exposition von „Romeo und Julia“, über, damit sie m mich verSTEHen. (Z. 115). Der erneute Strategiewechsel verdeutlicht das Scheitern der bisherigen Vermittlungsbemühungen.

ich $\mathrm{MACH}<<$ dim>mal $\mathrm{n}$ mal $\mathrm{n}$ BEIspiel;

115 damit sie m mich verSTEHen.>

116 (1.5)

117 ähm-

3 Das Beispiel ist transkribiert nach GAT (Selting et al. 1998). Der analysierte Transkriptausschnitt und der relevante Kontext finden sich im Anhang (Abschnitt 8). 


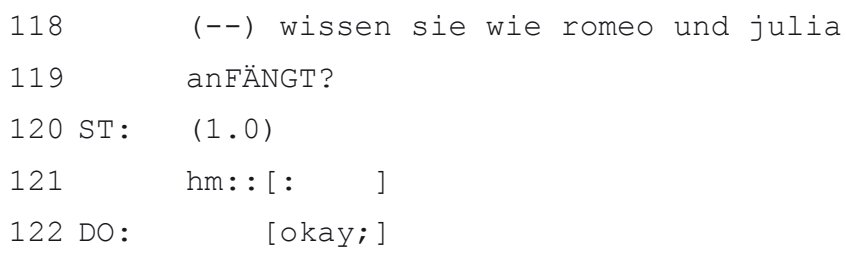

Die Beispielnarration beinhaltet zentral die multimodale Konstitution eines imaginären Raums. Bevor wir diese im Detail analysieren, fassen wir die interaktionsgeschichtlichen Bedingungen für ihr Zustandekommen kurz zusammen: Der Student hatte nicht auf die Vermittlungsbemühungen und die Angebote zum inhaltlichen Austausch reagiert und selbst keine Versuche unternommen, sein Verständnis von „Struktur“ zu verdeutlichen. Der Dozent setzt nun anstelle der bisherigen, zunächst abstrakten, dann zunehmend konkreter am Drehbuch des Studenten ansetzenden Fragen und Feststellungen, die auf den Gewinn der Selbsterkenntnis des Studierenden aus waren, die von ihm selbst vorgenommene Exemplifizierung als Vermittlungsverfahren ein.

Die Frage des Dozenten wissen sie wie romeo und julia anFÄNGT? (Z. 118119) bietet dem Studenten nochmals die Chance, sich selbstbestimmt mit der Dozentenkritik und seinem eigenen „Struktur“-Begriff auseinanderzusetzen. Als er auch sie nicht nutzt, startet der Dozent die Realisierung seines angekündigten Beispiels.

\section{Der imaginäre Raum der Exposition von „Romeo und Julia“"}

Im Folgenden wollen wir uns den Beginn der Exemplifizierung des Dozenten im Detail anschauen und uns dabei primär auf die Verfahren und Systematik konzentrieren, mit denen er den Raum konstituiert, den er für die Beispielrealisierung braucht. Wir wollen an dieser Stelle explizit darauf hinweisen, dass diese Raumkonstitution keine zwangsläufige Folge der Konstruktion eines Beispiels ist, sondern die Implikation der vom Dozenten gewählten Variante. Wir werden bei der Analyse auch immer danach fragen, in welcher Rolle der Dozent beim Aufbau und der Differenzierung seines Raumes/seiner Räume jeweils agiert und durch welche Mittel dies verdeutlicht wird.

\subsection{Eröffnung des imaginären Positionierungsraums}

Der Dozent beginnt die Beispielrealisierung unmittelbar mit einem Kernerzählsatz: 


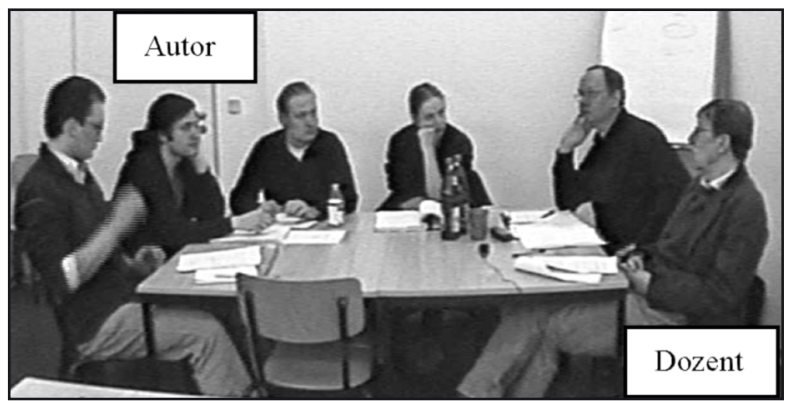

123 DO: da <<dim>GEHT ne GANG die STRABe runter; $>$

Wie die meisten Leser wissen werden (mit Sicherheit jedoch der Dozent weiß), fängt Romeo und Julia weder bei Shakespeare noch in der fraglichen Verfilmung mit der Szene an, die der Dozent mit seinem ersten Erzählzug etabliert. In beiden Fällen ist der Prolog, in dem die globale Struktur der gesamten Geschichte entworfen wird, dem faktischen Handlungsgeschehen vorangestellt. Ein Verweis auf den Prolog - und damit den tatsächlichen Anfang von Romeo und Julia - würde dem Dozenten jedoch bei seinem Versuch, dem Drehbuchautoren das Wesen der dramatischen Struktur exemplifizierend zu vermitteln, nicht nur nicht weiter helfen, sondern diesen sogar konterkarieren.

Die Tatsache, dass der Dozent den Prolog überspringt und sogleich mit der Darstellung der Handlungen der Protagonisten beginnt, ist eine erste empirische Evidenz für die reflexive Struktur seiner Beispielkonstruktion. Bereits der Beginn seines Beispiels reflektiert zentrale interaktionsstrukturelle Aspekte und Relevanzen der Situation „Pitching“: Die Studierenden lernen im Rahmen ihrer Ausbildung mit filmischen Mitteln eine Geschichte zu erzählen. Zentrale Voraussetzung dafür ist, ihre Protagonisten durch konkretes Handeln in ihrer wesentlichen Orientierung, ihren Zielen, Widersprüchen und Wünschen zu charakterisieren. Es geht gerade nicht darum, - wie dies im Prolog geschieht - sie in reflexiver, kategorialer und evaluativer Weise sprachlich vorzustellen oder zu beschreiben.

Das Drehbuch, welches das studentische Team vorgelegt hat, muss daher ohne die kontextualisierende Einführung eines Prologs auskommen bzw. die zentralen Informationen des Prologs handlungsmäßig und schrittweise etablieren. Um also die Vergleichbarkeit der studentischen Exposition mit der von Romeo und Julia zu maximieren und dadurch die Verständlichkeit der Beispielkonstruktion und ihre didaktische Funktionalität abzusichern, adaptiert der Dozent den Anfang des Dramas an die Struktur der studentischen Vorlage.

Während der Dozent in der Rolle des Erzählers sagt, dass eine Gang eine Straße hinuntergeht, behält er seine schon seit längerer Zeit statische 
Körperpositur bei: Er sitzt mit in seinem Schoß verschränkten Händen, die unter der Tischplatte ruhen, und in zurückgelehnter Haltung (Rücken an der Stuhllehne) am Tisch (Abb. 1).

Bereits in diesem ersten Erzählzug finden sich Aspekte, die raumkonstituierend sind:

$d a$

Für da (Z. 120) lassen sich zwei unterschiedliche Lesarten entwickeln:

1) Die erste Lesart begreift da als anaphorischen Bezug auf den Anfang von Romeo und Julia und damit primär als Hinweis auf die Exposition als aktuell zur Diskussion stehender dramaturgischer Sachverhalt. Das $d a$ wäre dann eine in der Form einer Raumreferenz verdichtete Reformulierung im Sinne „am Anfang von Romeo und Julia geht ne Gang die Straße runter".

2) Die zweite begreift da als raumdeiktisches Element. Es ist ein Verweis auf eine Räumlichkeit, die erst durch das, was noch in ihr passieren wird, nach und nach spezifiziert wird. Das da hat im Sinne von Goodwin (2003) die Qualität einer „projektiven Indexikalisierung“, die für eine nachfolgende Spezifizierung einen ersten, zunächst noch unstrukturierten Referenzrahmen etabliert.

die Straße

Eine Straße ist zweifelsohne ein komplexes räumliches Gebilde. Sie besitzt gewisse Ausmaße und Eigenschaften, die sie beispielsweise von einem Pfad oder einem einfachen Weg unterscheidet; sie ist zudem einer spezifischen architektonischen Umgebung assoziiert wie etwa einem Bürgersteig, Häusern (einem Straßenzug) und verweist damit auf einen öffentlichen sozialen Raum. Dies ist er mit keinerlei Zugangsbeschränkungen oder Sonderrechten für bestimmte Personen ausgestattet.

eine Gang

Eine Gang ist zwar selbst kein Raum, sondern eine soziale Gruppe, die jedoch durch eine spezifische Form des Raumbezuges charakterisierbar ist: Eine Gang besitzt und beansprucht Raum im Sinne eines eigenen Territoriums, in dem sie das Sagen hat, das sie beschützt, kontrolliert und gegen andere Gangs verteidigt.

runtergehen

„Runtergehen“ ist ein dynamisches, raumbezogenes Konzept, das eine zielorientierte Bewegung von A nach B in einem Raum mit einer gewissen Tiefe und Komplexität beschreibt. 
Die letzten drei Aspekte, die jeweils getrennt für sich betrachtet sehr unterschiedliche räumliche bzw. raumbezogene Implikationen haben, bilden zusammengenommen als geht ne Gang die Straße runter einen Raum, der in seiner Beschaffenheit und Struktur unweigerlich ein spezifischer sozialer Raum ist.

Dieser lässt sich nicht nur hinsichtlich seiner Relevanz für die Gang, sondern auch für diejenigen relativ präzise beschreiben, die der Gang begegnen. Es ist ein sozialer Raum, den die Gang durch eine spezifische kollektive Handlung (die Straße runter gehen) für ihre Selbstdarstellung benutzt. Wenn eine Gang die Straße runter geht, dann ist nicht primär die Überbrückung der Entfernung von A nach B der zentrale Punkt. Es geht vielmehr um eine spezifische Beanspruchung und In-Besitznahme des öffentlichen Raumes. Es handelt sich um eine aggressive Form der Selbstpräsentation, um eine bewusste Art, sich einem potenziellen Gegner zu zeigen, oder um die Einschüchterung derjenigen, die die Straße zufällig als Passanten benutzen.

Eine Gang, die die Straße hinuntergeht, konstituiert einen anderen sozialen Raum als die Krankenschwester oder die Reisegruppe, die die Straße hinunter gehen. Es ist zudem eine Form räumlicher Präsenz, die hinsichtlich der Formation, die die Gangmitglieder gemeinsam herstellen, charakterisiert werden kann: Diese werden keinen Gänsemarsch bilden, wie etwa Ausflügler aus dem Kindergarten, sondern die Straße möglichst „raumfüllend" beanspruchen. Es ist letztlich eine Form der Raumbeanspruchung, die Aggressivität symbolisiert und grundsätzlich auf Konfrontation ausgelegt ist.

Wir wissen zum jetzigen Zeitpunkt zwar welche Bewegungsrichtung die erste Gang hat, wir wissen allerdings (noch) nicht, wo genau sie sich auf der Straße bewegt: rechts, mehr in der Mitte oder links. Wir haben uns (auch weil der Sinn, wie sie noch sehen werden, von hinten kommt) für die - von der Origo des Sprechers aus gesehen - rechte Straßenseite entschieden.

Wir werden bei unseren folgenden Ausführungen die raumkonstituierenden Leistungen des Dozenten und die aufeinander folgenden Konstitutionsschritte in Form einer Skizze präsentieren. Die Skizzen sind nicht nur ein sinnvolles Medium der Visualisierung imaginärer Strukturen, sondern vor allem auch eine anschauliche Darstellung der sukzessiven Veränderungen des imaginären Raumes und seiner sich schrittweise verdichtenden und ausdifferenzierenden sozialen Struktur. Wir werden bei der Präsentation der Skizzen der Orientierung des Dozenten folgen und - soweit dies möglich ist - seiner grundlegenden Differenzierung und Abfolge der eingesetzten Verfahren der Raumkonstitution jeweils in einer eigenen Darstellung wiedergeben.

Wir werden nicht die gesamte Analyse auf dem bislang praktizierten detaillierten Explikationsniveau durchführen, sondern wollten gleich zu Beginn einmal exemplarisch auf die Reichhaltigkeit räumlicher Implikationen 
und die Permanenz impliziter Raumkonstitution allein durch das Medium Sprache verweisen. Wir wollen verdeutlichen, dass dies gerade auch dann der Fall ist, wenn Raumkonstitution nicht die primäre Aufgabe der sprachlichen Aktivitäten ist.

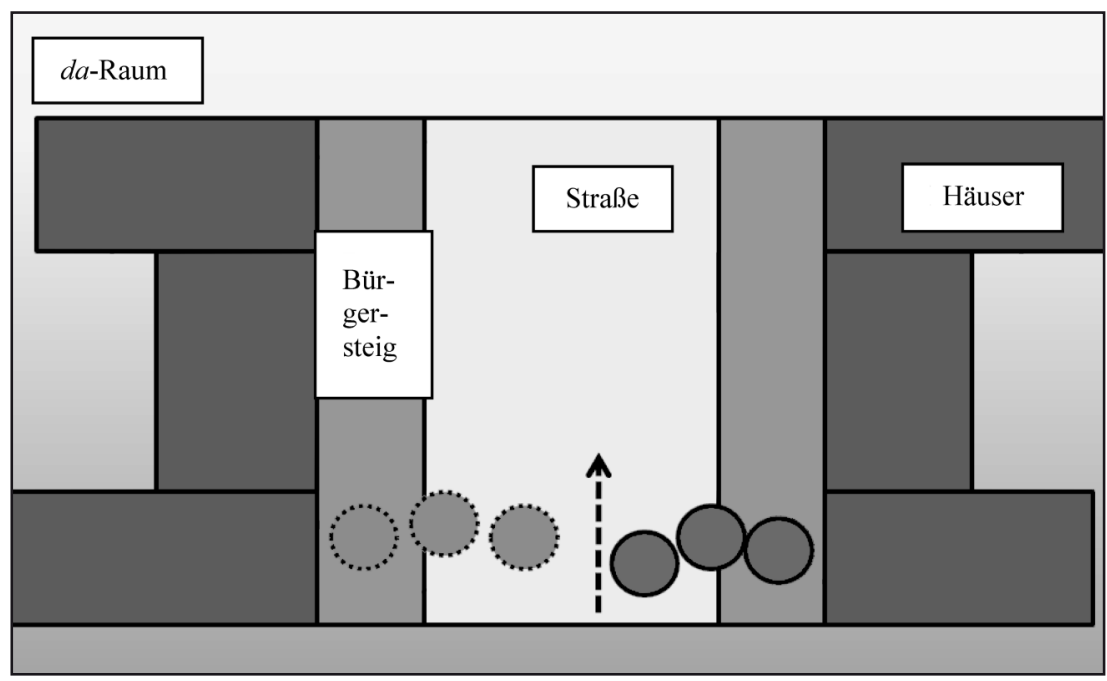

Skizze 1: Narrative Etablierung von Gang 1 im imaginären Raum

Legende:

$$
\begin{aligned}
& \mathbf{O}=\text { Gang } 1 \quad=\text { Alternativposition von Gang } 1 \\
& \hat{\mathbf{i}}=\text { Bewegungsrichtung (runtergehen) }
\end{aligned}
$$

Wir sind zudem nicht primär an der kaum hintergehbaren räumlichen Implikationen der Sprache an sich, sondern an dem Raum interessiert, den der Dozent als Bestandteil seiner alternativen Vermittlungsbemühungen im Kontext der Beispielkonstruktion explizit herstellt. Die kurze Explikation liefert jedoch folgende interessante Einsicht: Bereits in dem ersten kurzen Erzählzug liegt in impliziter Anlage die Struktur der Sozialität des Raumes als Potenzial und als eine fallspezifische Möglichkeit gegründet, die der Dozent im weiteren Verlauf seiner Beispielausführung herstellen und auch explizit verbalisieren wird. Schauen wir uns also die weitere Entwicklung des Beispiels und der Raumkonstitution an.

\subsection{Interaktionsstrukturierung und Verstehenssicherung}

$$
\begin{array}{ll}
124 \mathrm{DO}: & (-) \quad[j a ?] \\
125 \mathrm{ST}: & \quad[' \mathrm{mhm} ?]
\end{array}
$$




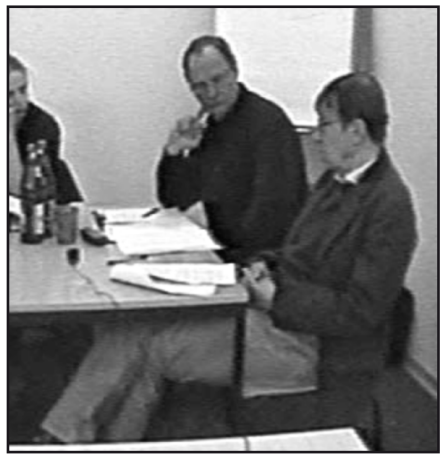

Abb. 2

$126 \mathrm{DO}: \quad(--)<<$ t $>$ ey-

Das ja? des Dozenten, das in Überlappung mit dem Rückmelder ' $m h m$ ? des Autoren realisiert wird, ist ein an den Studenten gerichtetes Vergewisserungssignal. Damit wechselt der Dozent aus der Rolle des Erzäblers in die des Interaktionsorganisators, der mit dieser verstehenssichernden Aktivität zum ersten möglichen Zeitpunkt in der Erzählung die didaktische Funktion seiner Darstellung verdeutlicht und den Studenten darüber hinaus zwingt, die bisher gelieferte Information explizit als verstanden zu bestätigen. Der Dozent realisiert damit auch einen räumlichen Wechsel. Er verlässt den mit da referenzierten Raum, in den hinein er die Gang positioniert hat, und agiert nun als Interaktionsmanager im gemeinsam mit den andern Anwesenden konstituierten Interaktionsraum. Wir haben es also mit zwei verschiedenen Räumen zu tun, in denen sich der Dozent in unterschiedlichen Beteiligungsrollen bewegt und die für die Bearbeitung seiner Vermittlungsanforderungen relevant sind: den Interaktionsraum und den imaginären Positionierungsraum.

Nach „erzwungener“ Rückmeldung des Studenten fährt der Dozent in der Entwicklung seines Beispiels fort, kehrt dabei jedoch nicht wieder zu dem mit da eröffneten Positionierungsraum zurück. Vielmehr eröffnet er einen dritten Raum: Es ist eine imaginäre Bühne, auf der er die zuvor positionierte Gang nunmehr mittels einer enaktierenden Darstellung in kategoriengebundener Weise präsentiert und vorführt.

Wir benutzen „Enaktieren“ im Folgenden zur Beschreibung eines interaktionsstrukturell motivierten, abhängigen sekundären Verfahrens. Die Abhängigkeit des Verfahrens zeigt sich formal betrachtet in seiner sequenziellen Nachordnung bzw. in der Vorgängigkeit eines relevanten Bezugsverfahrens. In unserem Fall wird Enaktieren systematisch eingesetzt zur Bearbeitung der durch eine vorgängige verbale soziale Kategorisierung (eine Gang) etablierten Anforderung nach situationsbezogener Spezifikation und Ausdifferenzierung der sozialen Kategorie. 
Es ist für Enaktieren konstitutiv, dass kontrastiv zur sprachlichen Kategorisierung noch andere Modalitäten als Ressource eingesetzt werden, die gerade nicht reflexiver, sondern demonstrativer Natur sind (Gestikulation, Blickverhalten, körperlich proxemisches Verhalten etc.). Man könnte auch sagen, dass - unter Bezug auf Schütz (1981) - situationsrelevante Aspekte des in der Kategorie sedimentierten sekundären Sinns, nahkontextuell und sequentiell nachgeordnet im Medium primärer Sinnkonstitution wieder aufgelöst werden. Man hat es letztlich mit einer Verfahrensbündelung bzw. einem Dualverfahren zu tun, das aus einer vorgängigen „sozialen Kategorisierung“ und einer nachfolgenden „Enaktierung“ besteht und das die zwei fundamentalen Modi der Sinnkonstitution in konvergierender Weise nutzt. ${ }^{4}$

\subsection{Eröffnung der Bühne}

\subsubsection{Auftritt der ersten Gang}

Mit diesem enaktierenden Zug seiner Beispielrealisierung verändert sich das Gesamtverhalten des Dozenten grundlegend. Er gibt seine statische Körperpositur und Handhaltung auf und wechselt in einen performativ-enaktierenden Modus.

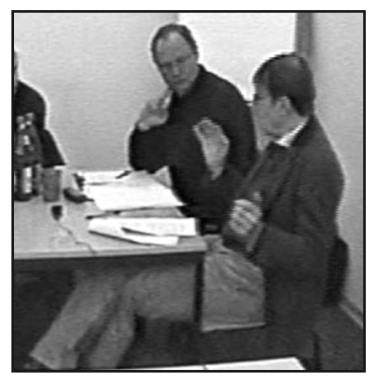

Abb. 3

126 DO: $\quad(--)<<t>$ ey-

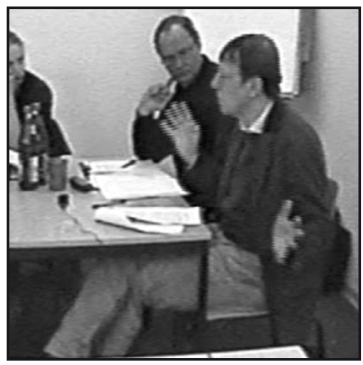

Abb. 4

\section{DO: (--) was S LOS alder;}

4 Enaktieren unterscheidet sich diesbezüglich von interaktionsstrukturell eher flexiblen, gesprächsrhetorischen Verfahren wie beispielsweise „Inszenieren“ (Schmitt 2003), mit dem es jedoch teilweise synonym verwendet wird. 


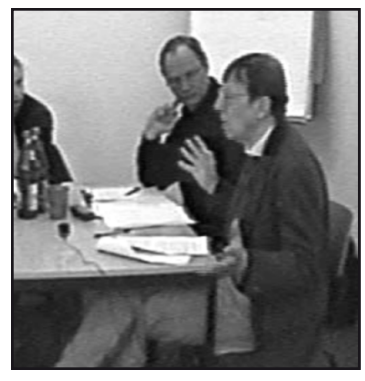

Abb. 5

128 DO: WO is randale; $>$

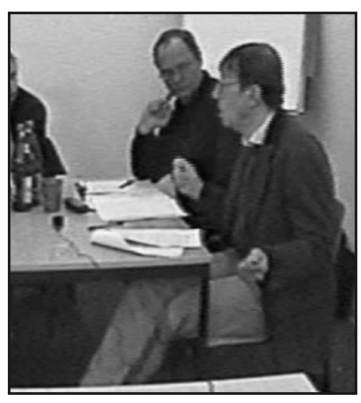

Abb. 6

129 DO: (-) nich?

$130 \mathrm{ST}:(-) \mathrm{mhm}$ ?

Seine Interaktionsbeteiligung ändert sich vor allem auf drei Ebenen, die wir im Folgenden im Auge behalten werden:

- die Stilistik des Sprechens,

- das körperliche Ausdrucksverhalten,

- die interaktive Beteiligungsrolle, in der der Dozent agiert.

Der Dozent geht dazu über, auf der Bühne als eines der Gangmitglieder zu agieren. Er spricht nun leiser, mit tieferer, knarrender, leicht gepresster Stimme. Es ist erkennbar nicht seine eigene Stimme, sondern er benutzt Jugendslang (Lexik: alder, randale; ey als attention getter, verschliffene Provokationsformel: was s LOS). Neben der verbalen und körperlichen Enaktierung des Verhaltens eines Gangmitglieds expliziert er mit wo is randale (Z. 128) auch dessen mentale Handlungsorientierung. Schon kurz vor Beginn dieses Turns schlüpft der Dozent in die Rolle des Gangmitglieds: Er gibt seine statische Oberkörperhaltung auf und löst beide Hände aus der Schoßhaltung, führt sie nach oben gut sichtbar über die Tischplatte und breitet Arme und Hände seitlich aus (Abb. 3-5). Seine Hände befinden sich nun rechts und links neben seinem Oberkörper, er spreizt alle Finger und bringt beide Handflächen nach vorne geöffnet in eine fast vertikale Position. Er schiebt dabei seinen Oberkörper, der mit dem Rücken an der Stuhllehne angelehnt 
war, etwas nach vorne. Mit Oberkörper und Kopf wippt er sechs Mal leicht nach rechts und links, wobei diese Bewegung auch die Unterarme und beide Hände integriert und teilweise noch leicht in seinem rechten Bein, das er über das linke geschlagen hat, sichtbar ist.

Diese Performance des Dozenten ist dominant von ihm aus nach vorne rechts, zum Drehbuchautoren hin, ausgerichtet. Er spricht nicht nur als Gangmitglied, er enaktiert auch dessen Gang durch die Stilisierung einer auf Wahrnehmbarkeit und Provokation angelegten, kategorientypischen Männlichkeitsdemonstration. Diese wippende Bewegung beendet der Dozent exakt mit einem erneuten Vergewisserungssignal nich? (Z. 129), das er deutlich lauter und höher spricht (Abb. 6).

Dadurch, dass der Dozent den Studenten erneut zur Verstehensdokumentation auffordert, verdeutlicht er, dass er mit dem ersten Zug seiner Inszenierung fertig ist. Zugleich schließt er die Finger beider Hände zu einer losen Faust und rückt sowohl mit dem Rücken wie auch mit dem Gesäß wieder in Richtung Stuhllehne. Die veränderte Finger- und Handgestik und die Rückkehr zur eigenen Stimme kontextualisieren den Übergang von der Enaktierung des Gangmitglieds zur Rolle des Lehrers und damit das Verlassen der Bühne und den Wiedereintritt in den gemeinsamen Interaktionsraum. Er nimmt seine Hände jedoch nicht wieder zurück in die home position (Sacks/Schegloff 2002), sondern bringt sie für alle sichtbar oberhalb der Tischplatte zur Ruhe, ohne sie jedoch dort abzulegen. Er kehrt auch mit seinem Oberkörper nicht vollständig in die Ausgangsposition zurück. Beides projiziert, dass der Dozent mit seiner Performance weitermachen wird.

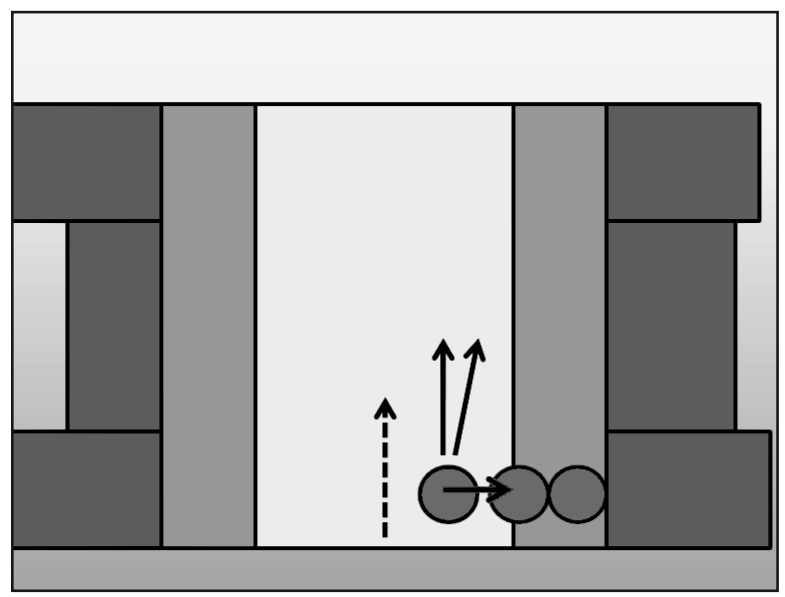

Skizze 2: Enaktive Positionierung Gang 1

Legende:

$\rightarrow=$ Adressierungsrichtung

$\uparrow=$ Blickrichtung 
Ziehen wir an dieser Stelle ein erstes Zwischenfazit:

- Wir können sehen, dass der Dozent in zwei unterschiedlichen Räumen - dem Interaktionsraum und dem imaginären Positionierungsraum und in verschiedenen Beteiligungsrollen agiert.

- Die Symbolisierung der Räume erfolgt

a) mittels spezifischer Sprechweisen, wodurch die Räume zu eigenständigen „Sprachräumen“ (Hausendorf in diesem Band) werden und

b) mittels spezifischer körperlicher Verhaltensweisen, die sich als besondere Form der Gestikulation und der Oberkörperbewegung und der Körper- und Blickorientierung zeigt.

- Auch die Performance als Gangmitglied und seine didaktische Strukturierung als Lehrer sind durch klare Grenzmarkierungen (Pausenklammer) und die punktgenaue Koordination zwischen den rollengebundenen Sprechweisen und den anderen Ebenen des multimodalen Ausdrucks konturiert und eindeutig voneinander abgegrenzt.

- Die Konstitution des imaginären Raumes weist zudem eine klare Sequenzialität auf: Zuerst wird der Raum narrativ strukturiert, dann wird in den etablierten Strukturen agiert.

\subsubsection{Auftritt der zweiten Gang}

Der Dozent führt nun parallel zur Darstellung der ersten Gang die zweite Gang ein. Erneut beschreibt er aus der Erzählerrolle das Auftauchen der zweiten Gang: da kommt ne ANdere gang (Z. 131). Wieder eröffnet er die Beschreibung mit $d a$, mit dem er die zweite Gruppe im gleichen Raum positioniert, in dem bereits die erste Gang unterwegs ist (vgl.: da $<<$ dim $>$ GEHT ne GANG die STRAße runter; > , Z. 123). Beide Gangs werden dann zueinander in Beziehung gesetzt, die zweite Gang adressiert die erste. Der Dozent wechselt nunmehr wieder seine Rolle und wandelt sich vom Erzähler zum Mitglied der zweiten Gang $\langle<h\rangle$ EY MANN DA geNAU (.) auf EUCH ham wir geWARtet; $>$ (Z. 132-133).

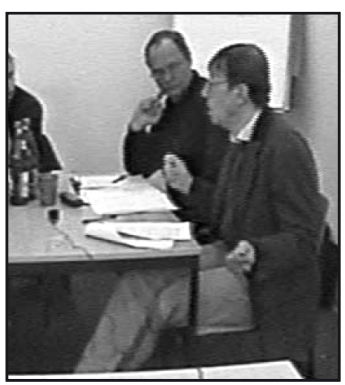

131 DO:

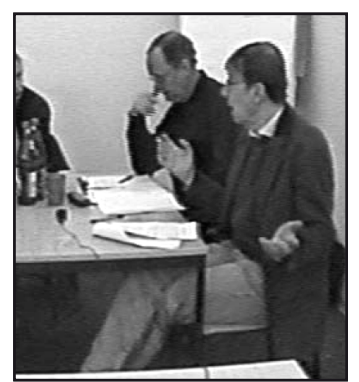

Abb. 7-8

ANdere gang und sagt 


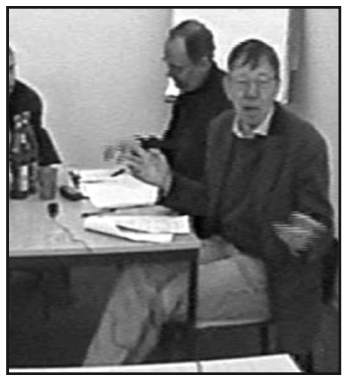

Abb. 9

$<<\mathrm{h}>$ EY MANN DA geNAU (.)

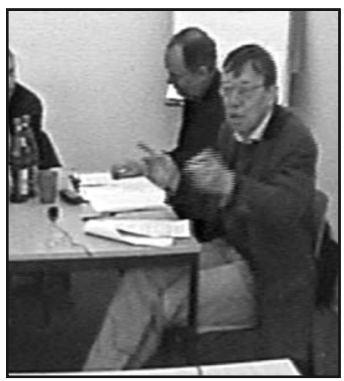

Abb. 10

$<<$ h $>$ EY MANN DA geNAU(.)

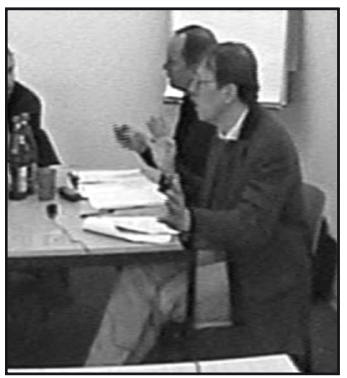

Abb. 11

133 DO: auf EUCH ham wir geWARtet; >

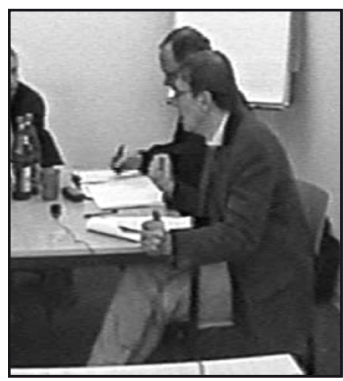

Abb. 12
$134 \mathrm{DO}$ :
(-) ja? (1.2)
135
(1.2) 
Wieder wird das Mitglied der zweiten Gang durch eine Sprechweise charakterisiert, die der ersten sehr ähnlich ist. Die Rede inszeniert er als dreiteiligen Turn, der strukturell dem des zuvor enaktierten gegnerischen Gangmitglieds sehr ähnlich ist:

- attention getter.

- 1. Formulierung der Provokation: was los alder - da genau

- 2. Formulierung der Provokation: wo is randale - auf euch baben wir gewartet

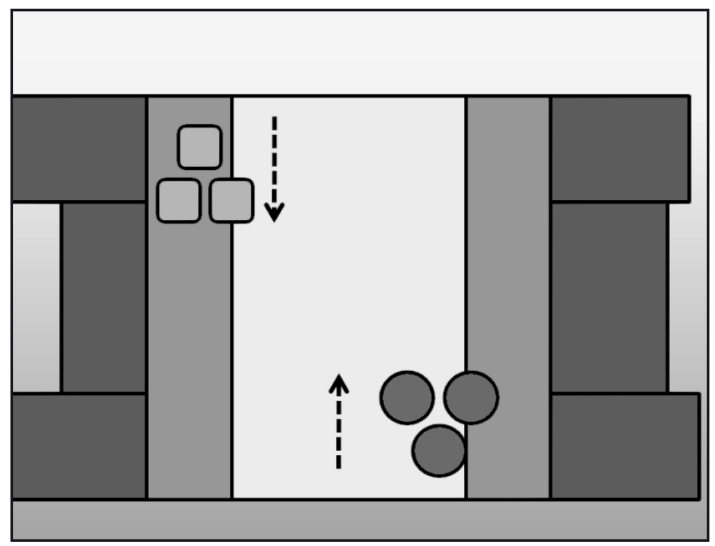

Skizze 3: Narrative Positionierung Gang 2

Legende:

$\square=$ Gang $2 \quad \dot{\mathbf{V}}=$ Bewegungsrichtung (kommen)

Anders als bei der ersten Gruppe steht im körperlichen Ausdruck jedoch nicht die Verkörperung des Gehens im Zentrum der Performance, sondern der Prozess des Suchens und Findens eines Gegners (Abb. 9-10). Dies verdeutlicht der Dozent durch die Drehung des Oberkörpers weit nach links, er lässt seinen Blick schweifen, deutet im Moment des Erkennens mit seiner linken Hand mit ausgestrecktem Zeigefinger in Richtung des Gegners, ehe er bei EUCH wieder zur Mitte zurückkehrt (Abb. 11), um seine Inszenierung der zweiten Gang abzuschließen. Analog zur Verdeutlichung der Position der ersten Gang im imaginären Raum, wird auch hier die Position der zweiten Gang durch den Blick nach links zur ersten Gang (auf der andern Straßenseite) markiert.

Wie bei der Darstellung der ersten Gang wechselt der Dozent, als er seine Enaktierung beendet hat, wieder in die Lehrerrolle: Er spricht wieder lauter und höher und ballt seine Finger zur Faust (Abb. 12). Wieder produziert er ein Vergewisserungssignal ( $j a$ ? ), das er mit einer kurzen Pause von seiner Performance absetzt. Der Dozent benutzt also die gleichen, ebenso 
systematisch und punktgenau multimodal und narrationsstrukturell koordinierten Verfahren der Enaktierung und des Wechsels unterschiedlicher Beteiligungsrollen.

Hatte er bei der performativen Darstellung der ersten Gang seine Enaktierung primär nach vorne-rechts ausgerichtet, so orientiert er sich nun bei der Einführung der zweiten Gang vorne-links. Die beiden Gangs sind nun also in einem Raum positioniert, in dem durch die körperliche Ausrichtung der Enaktierung und die sprachlichen Konzepte „,runter gehen“ und „kommen“ eine klare Opposition etabliert ist: Gang eins ist auf der einen, Gang zwei auf der anderen Straßenseite positioniert. Der im ersten Schritt aufgebaute imaginäre Raum wird weiter in Kraft gehalten und für die weitere Konstitution genutzt: Die zweite Gang entdeckt die erste Gang genau da im imaginären Raum, wo sie der Dozent positioniert und in Bewegung gebracht hatte. Der imaginäre Positionierungsraum ist dadurch nicht nur formal, sondern auch sozial und zeitlich strukturiert.

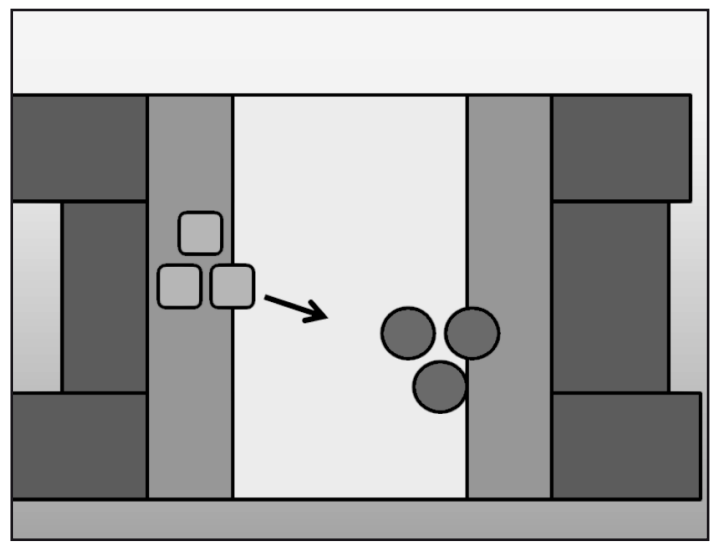

Skizze 4: Enaktive Positionierung Gang 2

Legende:

= Blick- und Adressierungsrichtung

Erst mit der Ausrichtung der enaktierenden Darstellung und der sprachlichen Adressierung der zweiten Gang ist auch die erste präzise in einem Rechts-Links-Schema verortet. Diese muss sich auf der rechten Seite (der Straße/des imaginären Raumes) befinden. Denn nur dann kann sie im Rahmen der durch die beiden Bewegungskonzepte „runter gehen“ und „kommen“ etablierte Perspektivität durch die nach links orientiere Bezugnahme der zweiten Gang adressiert werden. Hier kommt nun ein den vorliegenden Fall transzendierender, allgemeiner Aspekt zum Ausdruck, der für die Strukturierung und Perspektivierung imaginärer Räume insgesamt 
relevant ist. Die positionale Verdeutlichung handelnder Protagonisten in ihrer raumbezogenen Indexikalität muss nicht bereits mit dem Akt ihrer Positionierung mit erledigt werden. Vielmehr können diese Leistungen auch retrospektiv verdeutlichend erbracht werden: Die Positionierung der zweiten Gang zeigt dabei, wo sich die erste Gang befinden muss.

Der Vorteil einer solchen retrospektiven Positionierung besteht unter anderem in der damit zusammenhängenden Ökonomisierung der Raumkonstitution. Die Protagonisten können zunächst etabliert, charakterisiert und dann erst schrittweise durch die Orientierung beim Enaktieren in ihrer problembezognen Relationierung präzisiert werden. Man kann dabei aufgrund der Konstitutionslogik, die der Raumausgestaltung zugrunde liegt, präzise erschließen, wer wo platziert ist. Bei der Tatsache, dass die erste Gang von der zweiten als linksstehend indiziert wird, spielt die konkrete Seitlichkeit jedoch keine Rolle. Was relevant ist, ist vielmehr die präzise Modellierung der Konfliktstruktur durch die klare oppositive Positionierung der beiden Gangs.

Auf diese - gleichermaßen präzise und ökonomische Weise - wird genau das und genau so viel von der Konstellation narrativ etabliert und inszeniert, wie für die Funktionalität der Raumkonstitution als Verdeutlichung einer konkreten Konfliktstruktur nötig ist. Dass dabei ein Rechts und Links entsteht, ist nur wichtig als notwendige Voraussetzung für das „dazwischen gehen" des Sheriffs. Relativ zu diesen beiden Aspekten wird die Strukturierung des Raumes ausgestaltet. Es geht also nicht darum, die tatsächliche, komplexe und unübersichtliche Situation des Films abzubilden, sondern um die räumliche Darstellung einer idealisierten vereinfachten oppositiven Struktur. Bereits von Beginn an manifestiert sich, dass es nicht darum geht, anderswo existente Räume imaginierend ab- oder nachzubilden. Das didaktisch motivierte Ziel besteht vielmehr darin, einen imaginären Raum den spezifischen, durch die Interaktionsgeschichte motivierten pragmatischfunktionalen Zwecken gemäß zu modellieren und auszugestalten.

Es wird deutlich, dass wir es bei der im Imaginationsraum angelegten Konstellation nicht nur mit räumlichen Koordinaten zu tun haben. Es sind vielmehr unterschiedliche Protagonisten mit spezifischen, in diesem Fall korrespondierender sozialer Ausstattung und Orientierung, wodurch eine grundsätzlich räumlich-soziale Struktur entsteht. Der Imaginationsraum und das darin entworfene Geschehen besitzen darüber hinaus auch eine klare zeitliche Struktur. Die beiden Gangs befinden sich nicht nur in einem gemeinsamen Raum, sondern bewegen sich auch tatsächlich gemeinsam in diesem, was bedeutet, dass sie zum einen auch in einer gemeinsamen Zeit agieren und somit den Gesetzen der Sequenzialität unterworfen sind. Die zeitliche Struktur kann dahingehend präzisiert werden, dass die zweite Gang nicht sofort nach der ersten die Szene betritt. Vielmehr ist die erste Gang bereits eine Weile unterwegs, ehe sie auf die zweite Gang trifft. 
Nur durch die in der Zeit abgelaufene Bewegung der beiden Gangs (,runtergehen“ - „kommen“) ist es möglich, dass es zu der verbalen Adressierung kommt. Die beiden Gangs müssen sich im Laufe der Beispielkonstruktion sehr weitgehend angenähert und damit die potenzielle Konflikthaftigkeit (Randale, auf euch gewartet) der Situation verschärft haben.

\subsubsection{Konflikteskalation}

Nach der Enaktierung der beiden Gangs auf der Bühne ballt der Dozent beide Hände zu Fäusten (Abb. 12). Im Kontext des bislang inszenierten Aufeinandertreffens der beiden Gruppen verkörpert diese Haltung das narrativ bereits angelegte Konfliktpotenzial der Begegnung. Seine Entladung enaktiert er anschließend in einer gleichermaßen minimalistischen wie komplexen Weise.

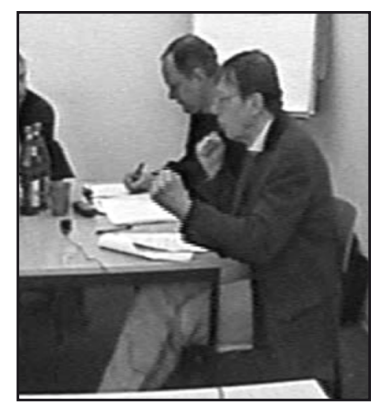

Abb. 13

136 DO: boff;

Der Dozent hebt beide Fäuste in die Höhe, führt im Stile eines Boxers seine linke Faust als Führhand etwas weiter nach vorne (zu einem imaginären Gegner) und hält die rechte als Schlaghand und zur eigenen Deckung zurück, etwas höher und näher an seinem Körper platziert. Während dieser Darstellung eines Boxers äußert er die Interjektion boff; (Abb. 13).

So klar diese Darstellung ein Emblem für die Konflikteskalation ist, so komplex ist sie in Bezug auf die Beteiligungsrolle, die der Dozent mit ihr einnimmt und hinsichtlich des Raums, in dem er dabei agiert. Er agiert in einem „Rollenmix“, der verschiedene Perspektiven miteinander vermischt. Verbale Äußerung und körperlicher Ausdruck ergänzen sich hier nicht wie zuvor in redundanter Weise zur Enaktierung einer einzigen Rolle. Sprache und körperliche Aktivitäten werden vielmehr lokal als autonome Ausdrucksmittel für die gleichzeitige Bearbeitung unterschiedlicher Aufgaben eingesetzt. Die an Sprechblasen in einem Comic erinnernde, onomatopoetische Interjektion boff bringt die Dynamik der Konflikteskalation zum Ausdruck und ist in diesem Sinne der maximal verdichtete Endpunkt der bislang narrativ entwickelten Struktur. 
Hans agiert dabei - was die Körperlichkeit der Darstellung betrifft (als Boxer) - auf der Bühne als eines der zum Kampf bereiten Gangmitglieder. Sprachlich hingegen kommentiert er diese Haltung, tritt also aus der Rolle des Boxers heraus und agiert als Erzähler, der einen auf einen Jugendlichen zugeschnittene metakommunikativen Kommentar realisiert.

Der Dozent agiert bei der Realisierung des boff auch wieder in unterschiedlichen Räumen: zum einen auf der Bühne, dem Platz der Enaktierung, zum anderen im Interaktionsraum, dem Platz vermittlungsspezifischer Verdeutlichungsleistungen. Die Perspektiven der Akteure, des Erzählers und Lehrers werden hier also in einem anschaulichen multimodalen Emblem des dargestellten Konfliktes kondensiert.

Darüber hinaus besitzt boff auch raumkonstituierende Implikationen. Dieser lautmalerische Ausdruck und die Boxerpose verdeutlichen nämlich, dass sich die beiden Gangs nicht mehr nur in Ruf-, sondern inzwischen auch in Schlagweite befinden. Sie haben sich inzwischen so weit angenähert, dass sie eine fokussierte Interaktion auf der Grundlage unmittelbarer körperlicher Kontakte starten können.

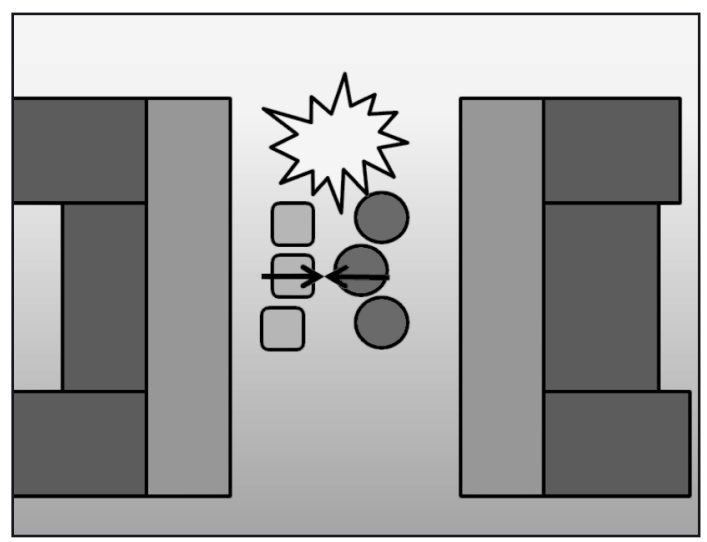

Skizze 5: Narrative/Enaktierte Positionierung der beiden Gangs (boff)

Legende:

$\longrightarrow \leftarrow=$,Verhaltensrichtung“ beider Gangs

Wir werden an dieser Stelle die detaillierte Rekonstruktion der Beispielentwicklung beenden. Da der Dozent bei seiner weiteren Entwicklung in systematischer und rekurrenter Weise die bereits beschriebenen Verfahren der Raumkonstitution und Indikation, der Charakterisierung der unterschiedlichen Protagonisten und die Symbolisierung seiner eigenen Beteiligungsrolle einsetzt, würde eine detaillierte Analyse über den aktuellen Punkt hinaus keinen wirklich neuen strukturellen Befund mehr zu Tage fördern.

Wir werden jedoch die weitere Entwicklung des Beispiels - soweit es für die Frage der Raumkonstitution wichtig ist - kurz beschreiben. 
Zunächst wollen wir jedoch die Gelegenheit zu einer zweiten Zusammenfassung nutzen.

Die bisherige Analyse bestätigt sehr weitgehend die Einsichten, die sich bereits im ersten Resümee gezeigt haben. Sie macht somit die Systematik und Rekurrenz der multimodalen Raumkonstitution des Dozenten deutlich:

- Es werden zwei unterschiedliche Räume konstituiert: der Interaktionsraum und der imaginäre Raum.

- In diesen beiden Räumen agiert der Dozent in drei verschiedenen Rollen: Im Interaktionsraum ist er Lehrer und Interaktionsstrukturierer; im imaginären Raum agiert er zum einen als Erzähler, zum anderen als Enaktierer.

- Die Positionen innerhalb dieser Räume werden systematisch durch unterschiedliche multimodale Konstitutionsverfahren gekennzeichnet.

- Erneut zeigt sich die klare sequenzielle Ordnung von

1) vermittlungsbezogenen Aktivitäten im Interaktionsraum,

2) narrativer Strukturierung des imaginären Positionierungsraumes und

3) enaktierender Verdeutlichung der Protagonisten dieses Raumes.

3.3.4 Namensgebung und Re-Positionierung

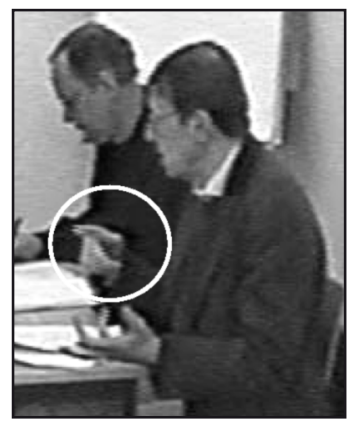

Abb. 14

137 DO: (--) die einen sind die CApulets,

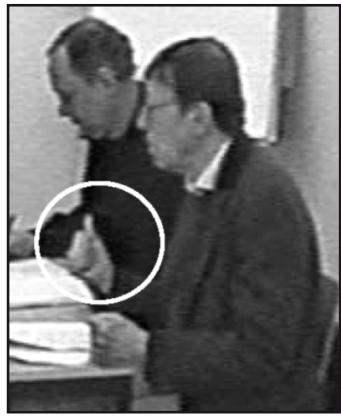

Abb. 15 
Als nächstes werden die Gangs namentlich vorgestellt (die einen sind die CApulets, und nach einer abermaligen kurzen Pause $<<\operatorname{dim}>$ und die anderen sind die DINGsens da; $>$. Die erste Gang, die Capulets, werden im Einklang mit ihren Koordinaten im Positionierungsraum mit dem gestreckten Zeigefinger der rechten Hand als links stehend ausgewiesen (Abb. 14). Die Dingsens (Montagues) werden dagegen - ebenfalls mit dem Daumen der rechten Hand - nach rechts verwiesen (Abb. 15). Der Dozent folgt also bei seiner Beispielentwicklung systematisch den von ihm selbst etablierten räumlichen Strukturen und verfestigt sie dadurch.

Im nächsten Schritt erfolgt der Auftritt des Sheriffs. Auch dabei wiederholt sich die Systematik von Etablierung im Positionierungsraum und der enaktierenden Darstellung auf der Bühne. Bei der Etablierung und der anschließenden Enaktierung des Prinzen orientiert sich der Dozent mit seinem Körper konsequent mittig nach vorne, während die Gangs rechts und links positioniert wurden. Diese Mittigkeit wird auch verbal durch das Konzept „dazwischen gehen“ formuliert.

140 DO: (--) und?

141 (--) wer geht dazWISCHen?

$142 \mathrm{ST}:(--) \mathrm{mh}$;

143 DO: (-) der SHEriff geht dazwischen;

144

der PRINZ geht <<all>dazwischen und sagt; >

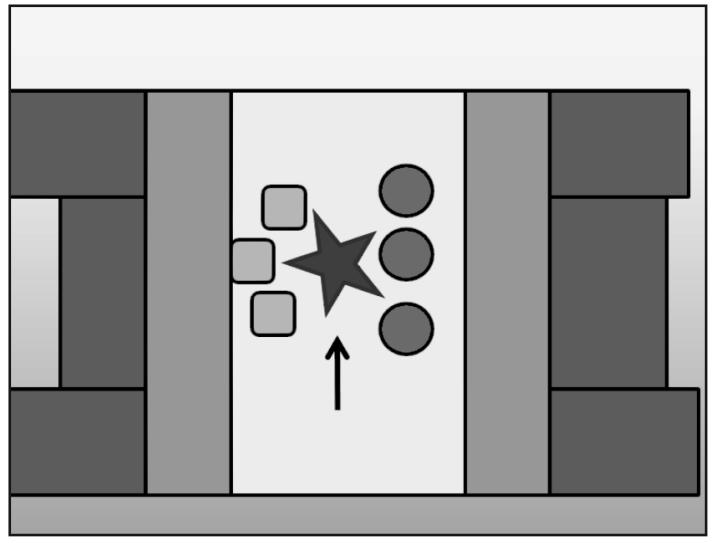

Skizze 6: Narrative Positionierung des Sheriffs/Prinzen (dazwischen gehen)

Der neue Akteur, der Sheriff, wird - im Unterschied zu den beiden Gangs, die beidhändig enaktiert wurden - durch die rechte Hand symbolisiert: Mit ausgestrecktem Zeigefinger und angehobenem Unterarm führt der Dozent zunächst eine Heranhol-Gestikulation und dann eine Mischung aus Zeigeund Mahngestikulation aus (Abb. 17-25, siehe unten). An dieser Stelle tre- 
ten erstmals in dieser Narration die verbale Einführung eines Akteurs und seine multimodale Enaktierung auseinander. Die Zeigegeste, die projektiv die folgende Warnung des Sheriffs verkörpert, setzt schon vor seiner verbalen Einführung ein; und bereits vor der folgenden Wiedergabe der ÄuBerung des Sheriffs verändert der Erzähler seine Stimme zunehmend in Richtung der Stimmgebung, die dann die folgende Rede des Sheriffs auszeichnet. Der Dozent wird langsamer, leiser und tiefer, die Stimme wird zur Knarrstimme. Stimme und Gestik sind diesmal nicht segmental kookkurrent mit den eingenommenen Rollen, beide antizipieren vielmehr die folgende Transition zwischen Erzähler und Geschichtenfigur.

Die gestikulatorischen Aktivitäten des Dozenten im Zusammenhang mit der Etablierung des Sheriffs lohnen eine etwas genauere Betrachtung. Dabei werden zwei Aspekte deutlich, die für unser auf Raum bezogenes Erkenntnisinteresse von Bedeutung sind: 1) die sequenzielle Ordnung der Gestikulation und 2) die Koordination der bei der Enaktierung eingesetzten modalen Ressourcen.

Ad 1): Die sequenzielle Ordnung der Gestikulation besteht aus zwei unterschiedlichen Teilen.

Der erste Teil ist eine Heranhol-Gestikulation, die mit gekrümmtem Zeigefinger realisiert wird (Abb. 16-21). Diese Gestikulation wird nicht simultan mit der enaktierenden Positionierung des Sheriffs/Prinzen realisiert, sondern erfolgt bereits bei dessen narrativer Etablierung. Es ist also noch nicht der Dozent als Sheriff, der diese Gestikulation ausführt.

„Heranhol“-Gestikulation
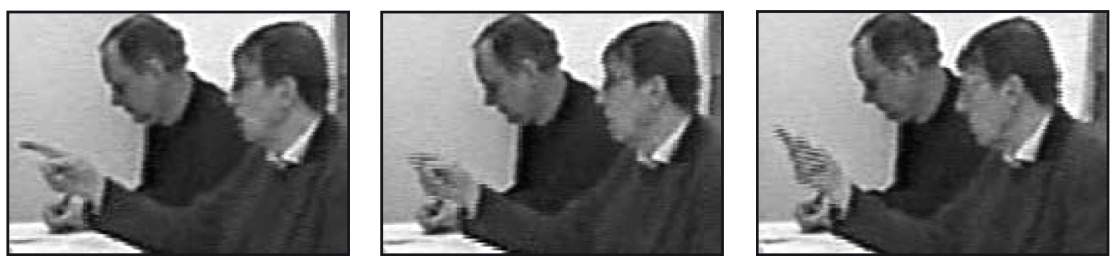

143 DO: (-) der SHEriff geht dazwischen;
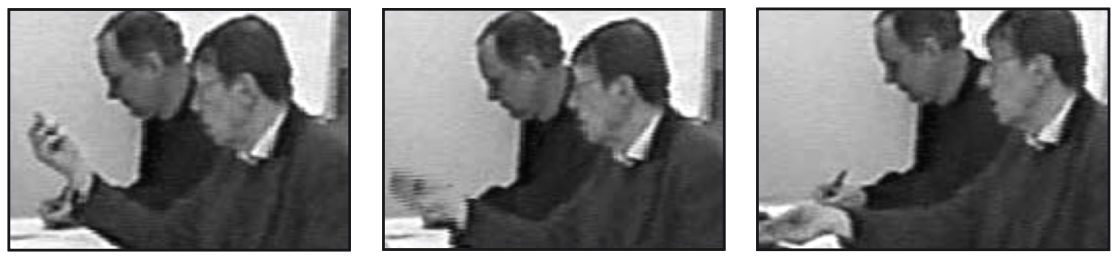

144

der PRINz geht <<all>dazwischen und sagt; $>$

Abb. 16-21 
Der zweite Teil ist eine Zeige- bzw. Ermahn-Gestikulation, die mit ausgestrecktem Zeigefinger ausgeführt wird (Abb. 22-24).
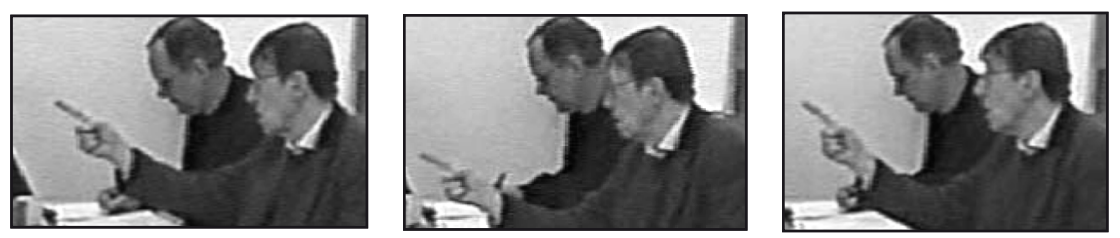

Abb. 22-24

Beide gestikulatorischen Segmente sind in erkennbarer Kontrastivität realisiert. Die Heranhol-Gestikulation ist dynamisch hinsichtlich der Höhe ihrer Ausführung und bezogen auf die Haltung der Finger und der Hand. Die Zeige- und Ermahngestikulation ist hinsichtlich der Finger- und Handhaltung hingegen statisch (der Zeigefinger bleibt die ganze Zeit über gestreckt und in die Höhe weisend). Lediglich die Ausführungshöhe variiert im persönlichen Verhaltensraum des Dozenten.

Ad 2:) Der zweite Aspekt ist die Koordination der gestikulatorischen Aktivitäten mit der verbalen Äußerungsproduktion. Die Koordination der sprachlichen Aktivitäten - vor allem in sprechrhetorisch relevanten Zusammenhängen der detaillierenden Charakterisierung der Agenten des imaginären Raumes und bei der Markierung der unterschiedlichen Beteiligungsweisen des Dozenten - war bislang durchgehend durch eine Systematik der Synchronität und des gemeinsamen Wechsels der beteiligten Modalitätsebenen charakterisiert. Hier an dieser Stelle jedoch tritt diese Systematik der Koordination zu Gunsten einer monomodalen Eigenständigkeit der gestikulatorischen Aktivitäten gegenüber den verbalen Aktivitäten des Dozenten zurück.

Die gestikulatorischen Aktivitäten erfolgen erkennbar und bedeutungstragend früher als die verbalen. Die Heranhol-Gestikulation ist vollständig „autonom“, d.h. sie begleitet nicht die Enaktierung, und auch der Beginn der Ermahn- und Zeigegestikulation liegt vor der verbalen Enaktierung des neuen Protagonisten. Durch diese zeitliche Vorgängigkeit erhalten beide Gestikulationen eine pragmatische Eigenständigkeit, die unabhängig von der Äußerungsproduktion des Dozenten in seiner Rolle als Sheriff/Prinz ist.

Dies hängt damit zusammen, dass sowohl das gestikulatorische Heranholen als auch die Zeige- und Ermahn-Aktivität im Interaktionsraum Adressierungsqualität besitzen. Der heranholende und der ausgestreckte und ermahnende Zeigefinger sind, genau wie der Blick des Dozenten, auf den Drehbuchautoren gerichtet. Diese Adressierung und die Vorgängigkeit der Gestikulation vor der enaktierenden Verbalisierung des Prinzen als neuem Agenten im imaginären Raum verleihen der Geste die zusätzliche Bedeutung, dass auch der Drehbuchautor ermahnt wird. Der Dozent realisiert hier also erneut eine Rollenmischung aus enaktierendem Erzähler und Leh- 
rer, agiert also sowohl im imaginären Raum als auch im Interaktionsraum. Dabei dient das gestikulatorische Heranholen gewissermaßen der Vorbereitung dieser Adressierungsdopplung: Der Drehbuchautor wird - metaphorisch gesprochen - zu den beiden Gangs hinzu geholt und dann mit diesen zusammen ermahnt.

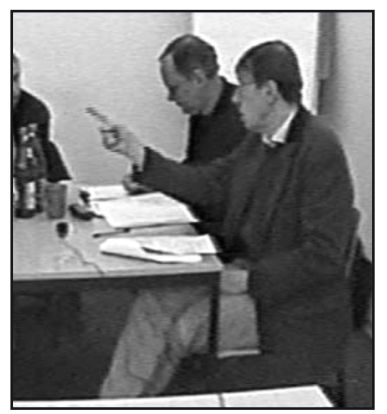

Abb. 25

$145 \mathrm{DO}: \quad<<\mathrm{p}>$ leute;

146 PASST mal auf;

147 (--) wenn das noch EI:Nmal passiert hier;

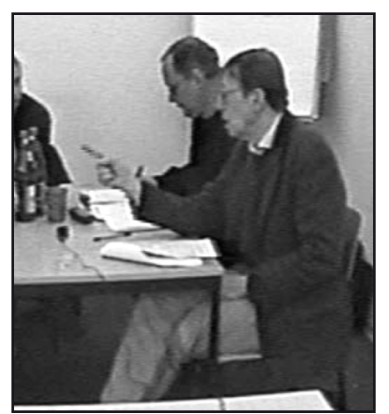

Abb. 26

148 DO: (-) dann-

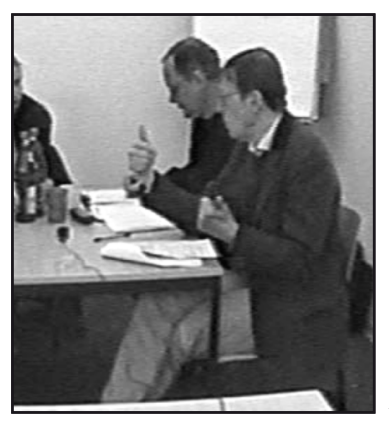

Abb. 27

(1.7)

150 DO: fliecht ihr hier aus der geMEINde; 


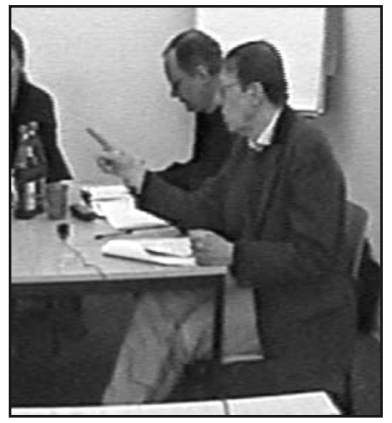

Abb. 28

151 DO: (-) dann werdet ihr vogelfrei;>

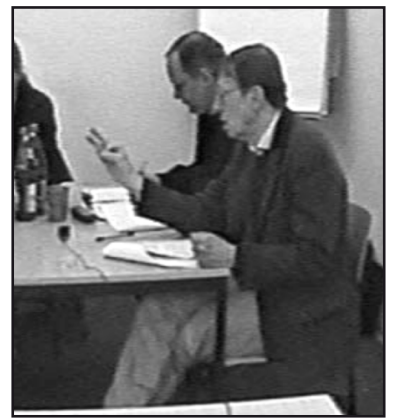

Abb. 29

$152 \mathrm{ST}:(---)$ ' $\mathrm{mhm}$;

153

(1.2)

Während die beiden Gangs sprachlich als Jugendliche enaktiert wurden, spricht der Prinz als Erwachsener und als Vertreter der Obrigkeit, die mit der Macht ausgestattet ist, den beiden Kontrahenten sehr weitreichende Konsequenzen anzudrohen. Die Stimme des Prinzen ist sehr weich und zunächst bedrohlich leise (bei $<<p>$ leute; PASST mal auf;), er hat keine Knarrstimme, sondern die Stimmbänder schwingen harmonisch.

Als letzter Schritt der Konstitution des Positionierungsraums deutet der Dozent bei der gestischen Darstellung des Rauswurfes, den der Sheriff den beiden Gangs androht, mit dem Daumen der rechten Hand nach rechts, mit dem Daumen der linken Hand nach links (Abb. 27). Der Rauswurf der beiden Gegner wird also relativ zu den etablierten Raumkoordinaten realisiert, die beide Parteien und der Prinz im imaginären Positionierungsraum einnehmen: Die Capulets fliegen nach links raus, die Dingsens (Montagues) nach rechts; beide von der Mitte aus gesehen, die der Prinz eingenommen hatte, als er dazwischen gegangen war (Abb. 30). 


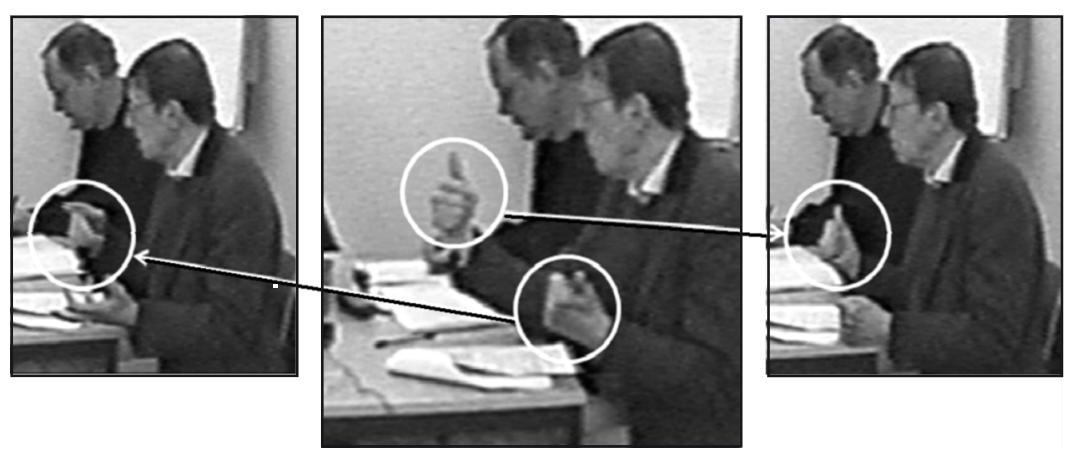

Abb. 30: Etablierung (links und rechts) und Rauswurf aus der Gemeinde (Mitte)

Die rechte und die linke Abbildung zeigen die gestikulatorische Verdeutlichung der räumlichen Positionen der beiden Gangs bei ihrer Einführung in den imaginären Raum: links die Capulets (siehe Abb. 14), rechts die Dingsens (Montagues) (Abb. 15). Die mittlere Abbildung verdeutlichte die positionale Konstanz der beiden Gangs im imaginären Raum zum Zeitpunkt des angedrohten Rauswurfs: Jede Gang fliegt auf ihrer Seite aus der Gemeinde.

\section{Rückführung zum kategorial-abstrakten Vermittlungsdiskurs}

Nachdem der Dozent die Erzählung der Ereignisse der Exposition abgeschlossen hat, verdeutlicht er zusammenfassend die dramatische Struktur der von ihm gerade erzählten und enaktierten Exposition:

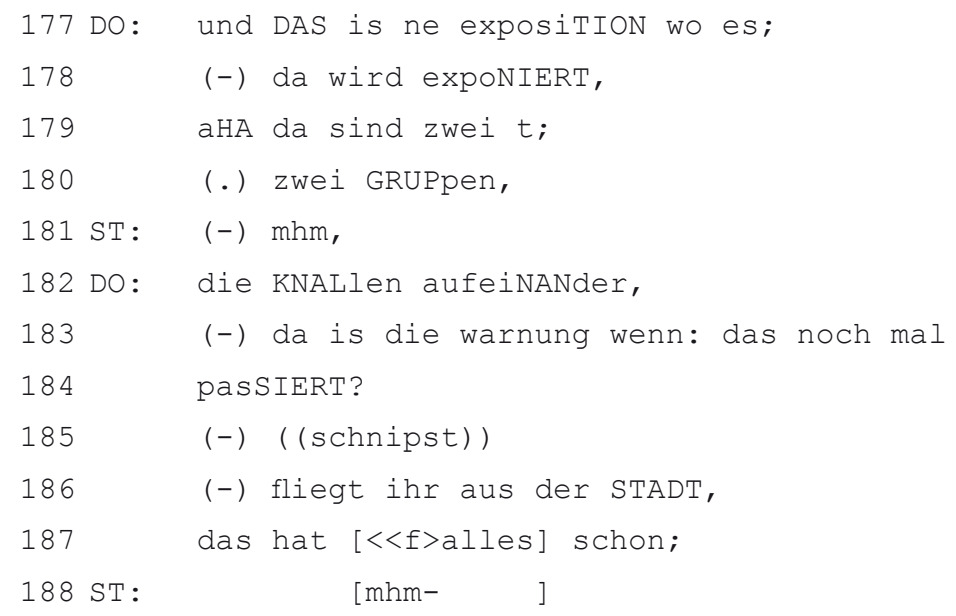


189 DO: GANZ> unmittelbar was;

$190 \quad(-) \quad<$ dim>MIT <<dim>unserer geschichte

191

zu tun; $>$

Mit dieser Strukturverdeutlichung macht der Dozent nochmals klar, worin die besondere dramaturgische Qualität seines Beispiels besteht: Jedes Element der Exposition hat eine unmittelbare Relevanz für die Handlung, die sich im Verlauf des Dramas entfalten wird. Die interaktive Funktion dieses Beispiels arbeitet er weiter heraus durch die folgende negativ evaluative Kontrastierung mit dem studentischen Drehbuch:

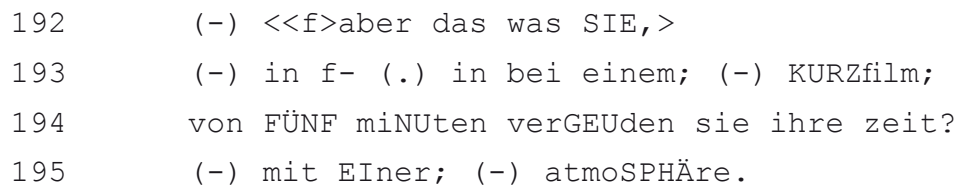

Mit der zu exposiTION (Z. 177) kontrastierenden, abgewerteten Kategorie atmoSPHÄRE rekontextualisiert er die von ihm abgelehnte Kategorie des Studenten; er bezieht seine Ausführungen somit zurück auf den Ausgangspunkt der Beispielnarration. Der Dozent verdeutlicht rückwirkend, dass seine narrative Performance einem argumentativen Zweck diente, nämlich die Unzulänglichkeit der Exposition des studentischen Drehbuchs durch den Vergleich mit einer gelungenen aufzuweisen und dadurch die Irrelevanz von atmoSPH ÄRE als dramaturgischer Kategorie zu verdeutlichen.

\section{Spezifik der Raumkonstitution als „Problemlösung“}

Betrachtet man das, was der Dozent in dem von uns analysierten Ausschnitt macht, im konversationsanalytischen Verständnis als Lösung interaktiver Probleme, so lassen sich die folgenden Problemaspekte angeben, als deren Lösung die rekonstruierte Spezifik der Raumkonstitution im interaktionsstrukturellen Kontext entwickelt wird.

Damit der Dozent im Rahmen seiner alternativen Vermittlungsbemühungen (ein raumbasiertes Beispiel mit konkret handelnden Agenten, die in ihrer spezifischen Qualität enaktierend vorgeführt werden, um damit die dramaturgische Funktionalität und Struktur einer Exposition zu verdeutlichen) Raum als Ressource überhaupt nutzen kann, muss er

a) Raum als relevante Größe etablieren,

b) die Positionierung, Relationierung und soziale Kategorisierung der Agenten, die im etablierten Raum/den etablierten Räumen handeln und aufeinander stoßen, aufzeigen,

c) verdeutlichen, in welchem Raum er sich gerade befindet, 
d) seine raumspezifische Beteiligungsrolle verdeutlichen,

e) die Beziehung, der unterschiedlichen Räume untereinander aufzeigen und

f) die Funktionalität, die die Räume für die Konstruktion des Beispiels besitzen, verdeutlichen.

Wir werden uns bei unserer weiteren Reflexion der Analyseergebnisse primär um die schrittweise Etablierung und Aufrechterhaltung des da-Raumes kümmern, den wir als Positionierungsraum bezeichnen. Wir werden dabei vor allem die Positionierung der verschiedenen Agenten des Raumes, deren Relationierung und Kategorisierung sowie die Beziehung des Positionsraumes zur „Bühne“ und zum „Interaktionsraum“ in den Fokus nehmen.

\section{Die Multidimensionalität von Raum in der Interaktion}

Nachdem in der Fallanalyse gezeigt wurde, wie verschiedene Räume durch unterschiedliche Verfahren konstituiert und markiert werden, möchten wir nun explizit die verschiedenen Dimensionen von Raum, die im Beispiel relevant werden, differenzieren und ihr Verhältnis zueinander klären. Die Literatur zur Raumkonstitution und Deixis geht in der Tradition Bühlers in Beispielen wie dem unsrigen von zwei Räumen aus: dem Wahrnehmungsraum („,demonstratio ad oculos“, Bühler 1934, S. 80 ff.) und dem Vorstellungsraum (Deixis am Phantasma; vgl. etwa Bühler 1934, S. 125 ff.; Fricke 2007; Sitta 1991). In Anknüpfung an neuere Untersuchungen zur Raumkonstitution in der multimodalen Interaktion (z.B. Goodwin 2003; Mondada 2005; 2007) müssen wir jedoch von einer komplexeren Konstellation mehrerer aufeinander bezogener Räume ausgehen, welche die dynamische Räumlichkeit der sozialen Interaktion als solcher zentral in der theoretischen Konzeption von ,Raum` mitberücksichtigt. Vier Räume lassen sich differenzieren:

1) Der Raum als sozio-physische Umgebung

2) Der Interaktionsraum

3) Der individuelle Verhaltensraum

4) Der imaginäre Raum

\subsection{Raum als Umgebung}

Hiermit meinen wir den physikalischen, territorial-architektonischen und sozial kodierten Raum. Dies ist der Raum, wie er unabhängig von einer in ihm stattfindenden Interaktion von einem Beobachter zu beschreiben wäre. Dieser Raum ist eine physikalische Größe mit Tiefen- und Breitendimensionen, physikalischen Begrenzungen und enthaltenen Körpern, die in so- 
zialer Beschreibung durch seine innenarchitektonische Ausstattung gegeben sind. Definiert wird der Raum(ausschnitt, den die Kamera zeigt,) durch die zusammengestellten Tische, die einen großen Tisch bilden und die besondere Anordnung der Stühle, die zu einer bestimmten Konstellation der Interaktionsbeteiligten führt.

Dieses physikalische Arrangement ist sozial kodiert und hat dadurch spezifische Implikationen für die Interaktionen, die in ihm stattfinden können. Zu diesen gehört, dass man in diesem Raum Aktivitäten erwarten kann, die sich relativ eng um den Tisch herum entfalten werden. Die TischStühle-Konstellation verweist auf eine dominante usuelle Funktionalität, für die der Raum ausgestattet worden ist: Es geht um die primär sprachliche Kooperation mehrerer Personen im Sitzen. Darüber hinaus vermittelt das Arrangement als weiteren erwartbaren Aspekt der Interaktion eine gewisse Statik und Konstanz nicht nur der Positionen und Präsenzformen der Interaktionsbeteiligten, sondern auch der Kooperationsform die sie gemeinsam praktizieren werden. „Raum als Umgebung“ beinhaltet also physikalische und soziale bzw. symbolische Aspekte, die jedoch allein schon deskriptiv kaum klar zu differenzieren sind. Eine theoretisch denkbare Subdifferenzierung eines physikalischen von einem sozial(symbolisch)en Raum ist daher für unsere Zwecke nicht sinnvoll. Der entscheidende Punkt für unsere Systematik ist vielmehr, dass Räume aufgrund ihrer physikalischen Bedingungen und aufgrund sozialer Codierungen Restriktionen, Möglichkeiten und Erwartungen mit sich bringen, die unabhängig von der in ihnen stattfindenden Interaktion (in einer Kultur) für die Interaktanten gelten und sich auf die in ihm stattfindenden Interaktionen auswirken (vgl. Goffman 1961).

\subsection{Raum als Interaktionsraum}

In ihrer Interaktion konstituieren die Beteiligten aus dem Umgebungsraum ein für die Zwecke des gemeinsamen Handelns konstituierten sozialen Relevanzrahmen, den wir als „Interaktionsraum“ bezeichnen (siehe Schmitt/ Deppermann 2007; Mondada 2007). Der Interaktionsraum hat eine soziophysische Dimension, die Beteiligungsstruktur (participation framework: Goffman 1981; Goodwin/Goodwin 2004), die in den proxemischen Relationen und positionalen Strukturen der Beteiligten besteht (siehe auch Kendon 1990). Der Interaktionsraum ist stets ein orientierter Raum: Die Beteiligten sind aufeinander und ggfs. auf spezifische Wahrnehmungsfoki als Teil dieses Raums bezogen. Diese Orientierung konstituiert sich durch die leibliche Ausrichtung der Interaktanten (z.B. face-to-face, side-by-side, body torque; Schegloff 1998; Proxemik, Blickkontakt) und ihren Bezug auf gemeinsame Wahrnehmungsobjekte und Demonstrata, die durch die Interaktion selbst (z.B. durch Blickrichtung, Zeigen, Deixis) relevant gemacht werden. Der Interaktionsraum ist ein prozessualer, aktivitätskonstituierter Raum, der nicht un- 
abhängig vom Handeln der Beteiligten „in ihm“ existiert (siehe Schmitt (Hg.) 2007). Die Herstellung und Veränderung des Raums ist pragmatisch durch die Kooperation in Bezug auf gemeinsame Handlungsaufgaben, die mit ihnen zusammenhängenden Beteiligungsweisen der Einzelnen und ihre in diesem Kontext relevanten räumlichen Orientierungen bestimmt.

\subsection{Raum als individueller Verhaltensraum}

Individuelle Verhaltensräume sind unter einer sozialen Perspektive personenzentrierte Teilbereiche des Interaktionsraumes. Sie können charakterisiert werden als individuelle, sozial-territoriale Umgebung von einzelnen Interaktionsbeteiligten (Goffman 1963). Obwohl personal gebunden, werden auch die individuellen Verhaltensräume letztlich durch gemeinsame - eigene und fremde - Definitionsleistungen konstituiert. Zu diesen Definitionsleistungen gehören beispielsweise proxemische Aspekte wie Positionierung zu den anderen Interaktionsteilnehmern (vgl. Hall 1966; Scollon/Wong Scollon 2003), die Körperpositur sowie die gestikulatorischen und in unserem Fall sehr prägnant auch die enaktierenden Aktivitäten des Akteurs. ${ }^{5}$ Die Begrenzung(en) individueller Verhaltensräume werden teilweise durch symbolische Grenzziehungen markiert. Hierzu zählen beispielsweise das Arrangement der Arbeitsutensilien, abgestellte Gläser und Flaschen etc. Diese individuellen Verhaltensräume werden (mit-)konstituiert durch korrespondierende oder konkurrierende Orientierung und Respektierung der anderen Beteiligten. Individuelle Verhaltensräume sind wie Interaktionsräume, da sie an die Körperlichkeit der Interaktionsteilnehmer gebunden sind, grundsätzlich dynamisch - und in unserem Fall nicht unbedingt an die Sitzposition gebunden. Durch Aufstehen und Bewegung im Raum können individuelle Verhaltensräume modifiziert und erweitert werden.

\subsection{Raum als imaginärer Raum}

Unter der für Interaktionsräume konstitutiven Bedingung von Wahrnehmungswahrnehmung (vgl. Hausendorf 2003) können Teilnehmer mit ihren multimodalen Aktivitäten imaginäre räumliche Konfiguration entwerfen und diese Räume durch ihre Aktivitäten ausgestalten und für eine gewisse Zeit aufrecht erhalten. Mit „imaginären Raum“ meinen wir hier räumliche Strukturen, die in der Interaktion symbolisch konstruiert werden und auf die referiert wird, die aber für die Interaktionsbeteiligten in der Interaktionssituation nicht visuell wahrnehmbar sind. Der imaginäre Raum kann empirisch nicht als mentaler Raum untersucht werden, sondern nur in Bezug auf seine Verfahren der kommunikativen Konstitution. Deshalb benutzen wir hier nicht den gängigen mentalistischen Begriff „Vorstellungsraum“

Vgl. auch die Konzeption des „gesture space“ bei McNeill (1992, S. 89). 
(Bühler 1934, S. 125 ff.), sondern den Begriff „imaginärer Raum“. Imaginäre Räume sind zeichenkonstituiert (vgl. Fricke 2007, S. 87, 128 ff., 249 ff.). Ihre Herstellung erfordert interaktive Arbeit, denn grundsätzlich zeichnen sich imaginäre Räume durch eingeschränkte Dauerhaftigkeit und ephemere Objekthaftigkeit aus, außer wenn sie beispielsweise qua Zeichnung objektiviert sind (vgl. die Tabelle in Fricke 2007, S. 257). Imaginäre Räume werden in Bezug auf die pragmatischen und thematischen Anforderungen der Interaktion konstituiert und stellen einen Beitrag zu deren Bearbeitung dar.

\section{Multimodale Verfahren der Konstitution von imaginären Räumen}

\subsection{Die schrittweise Konstitution des imaginären Raums}

Die Schaffung und Ausgestaltung eines imaginären Raums geschieht in drei systematischen Schritten:

a) Die Raumkonstitution nimmt ihren Ausgang im Interaktionsraum, aus dem heraus der imaginäre Raum etabliert wird.

b) Der imaginäre Raum wird narrativ durch Erzählsätze konstituiert, in unserem Fall durch den orientierenden Satz da geht ne gang die straße runter. ${ }^{6}$ Der Sprecher ist hier also Erzähler im Interaktionsraum.

c) Die Origo des Sprechers wird nun in diesen narrativ geschaffenen Raum versetzt, in unserem Beispiel in die erste Gang, die als erste in diesen Raum positioniert wurde. Der Sprecher ist nun die Figur, die im imaginären Raum enaktiert wird. Die gleiche Abfolge von erstens narrativer Einführung der Figur und ihrer räumlichen Positionierung im imaginären Raum und zweitens Versetzung der Sprecher-Origo in diese Figur durch Enaktierung wiederholt sich bei der zweiten Gang und dem Sheriff/Prinz. Wir sehen also, dass (wie dies auch Fricke 2007, S. 70 und 87 f. feststellt) die Konstitution des imaginären Raums die unabdingbare Voraussetzung für die anschließende Versetzung der Origo in diesen ist.

In der Literatur wird immer wieder hervorgehoben, dass das Vokabular der Räumlichkeit die wichtigste Quelle für das Vokabular der Zeitlichkeit sei. ${ }^{7}$

Im Sinne von Labov/Waletzky (1973) handelt es sich um einen orientierenden Satz, der das narrative Geschehen grob situiert, zugleich aber auch schon ein Kernerzählsatz ist, da er eine erste komplikationsrelevante Handlung beschreibt.

7 Diese linguistische Fundierung von Zeitlichkeit in Räumlichkeit wird sowohl in Bezug auf die Sprachgeschichte bzw. Etymologie (Hopper/Traugott 2003, S. 84-87; Haspelmath 1997), als auch in Bezug auf die individuelle Sprachentwicklung (Clark 1973) und die mentale Konzeptualisierung im Sinne von ,Raum`als root metaphor für ,Zeit' (Lakoff/Johnson 1980) postuliert. 
Bei der Raumkonstitution in der Interaktion sehen wir ein umgekehrtes Fundierungsverhältnis: Räumlichkeit kann sich nur in Zeitlichkeit entfalten. Es ist unmöglich, eine komplexe räumliche Konstellation monothetisch (wie Husserl schrieb) zu kommunizieren, man muss sie in polythetischen, d.h., einzelnen aufeinander folgenden Akten entfalten. Unser Fallbeispiel hat gezeigt, dass dafür nicht nur die strukturelle Latenz, d.h., die fortdauernde Geltung und mentale Verfügbarkeit von Äußerungen, die auf Räumliches Bezug nehmen, eine Voraussetzung ist. Das Gleiche gilt auch für Gesten und Enaktierungen: Nur auf dem Hintergrund einer vorangehenden Gestikulation und Enaktierung gewinnt eine folgende ihren relationalen Sinn als Schritt und Komponente der weiteren Ausgestaltung eines komplexen räumlichen Szenarios. Ihre räumliche Bedeutung ist sequenzabhängig. So partizipieren etwa in unserem Beispiel die Deutung der Geste und die lexikalische Referenz des „Dazwischengehens“ (des Sheriffs/Prinzen) am aufgebauten Referenzrahmen der physischen Konfrontation der Gangs, durch den sie eine literale und nicht bloß metaphorische Interpretation im Kontext des imaginären Raums gewinnen. Diesen Rahmen selbst können Geste und Raumvokabular aber nicht gleichzeitig mit ihrer Produktion konstituieren. Wie wir bei der Enaktierung der zweiten Gang und ihres Verhältnisses zur ersten sehen, funktioniert diese interpretative Anreicherung nicht nur prospektiv im Sinne einer zunehmenden Spezifikation durch neue Elemente. Sie kann genauso retrospektiv funktionieren, indem nämlich die Positionierung eines neuen Elements (hier: eines Akteurs) im Raum ein schon früher eingeführtes Element in seiner räumlichen Position und Dynamik genauer bestimmt. So wird im Beispiel die Position der ersten Gang auf der Straße erst im Nachhinein, durch die auf sie bezogene Positionierung der zweiten Gang klar. In diesen Hinsichten ist die Zeitlichkeit der Interaktion Voraussetzung für die Konstitution von Räumlichkeit.

Doch Zeitlichkeit ist wenigstens in unserem Beispiel noch in einer anderen Weise an der Konstitution von Räumlichkeit beteiligt. Der Dozent staffiert nicht nach und nach eine statische Situation aus, sondern er entwirft ein dynamisches Szenario, in dem die räumlichen Verhältnisse gerade durch ihre Veränderung Kontur gewinnen: Auch die statischen „Momentaufnahmen" der Verhältnisse im Interaktionsraum gewinnen nur durch die Darstellung von sie verändernden Handlungen Kontur, so z.B. im boff (Z. 136), das mit der Darstellung der physischen Konfrontation zugleich die räumliche Position der Gangs zueinander neu bestimmt.

\subsection{Modalitätsspezifik der Raumkonstitution}

Die Schaffung und Ausgestaltung eines imaginären Raums entsteht durch den Einsatz unterschiedlicher, systematisch miteinander verknüpfter Verfahren. Die initiale verbale Konstitution eines nicht präsenten Raums durch 
eine Beschreibung ist die Konstitutionsgrundlage für alle weiteren Schritte (siehe 6.2). Dieser Raum wird durch verbale und gestische Ressourcen schrittweise weiter ausgestaltet. Er wird dabei nicht nur narrativ konstituiert, sondern auch enaktierend zu einer Bühne verwandelt, auf der der Dozent nunmehr die Origones der Figuren in diesem Raum verkörpert. In unserem Beispiel werden für die Konstitution des imaginären Raums nicht nur räumliche Deixis und Zeigegesten benutzt, sondern weitere Verfahren, die jeweils eine ganz spezifische Funktion innerhalb des Gesamtzusammenhangs der Raumkonstitution haben:

a) Raumvokabular: da, kommt, runtergehen, dazwischengehen und rausfliegen sind Ausdrücke, die räumliche Verhältnisse relational bzw. deiktisch repräsentieren und die perspektivisch auf die Origo des Konzeptualisierers der Szene bezogen sind (vgl. Fillmore 1997). Das räumliche Vokabular im Beispiel situiert das Geschehen in der Nähe-Distanz-Dimension: Es ist so ausgewählt, dass mit ihm der Prozess der räumlichen Annäherung der Gangs und ihrer Trennung durch den Sheriff/Prinz dargestellt wird.

b) Zeigegesten im imaginären Raum sind im Beispiel die Geste des Entdeckens der ersten Gang durch die zweite, die Geste des Prinzen, der auf die Gangs ermahnend zeigt und die Zeigegesten des Rausfliegens nach rechts und links. Diese Gesten situieren die Figuren im imaginären Raum in einer Links-rechts-Dimension, die orthogonal zur sprachlich realisierten Dimension der räumlichen Annäherung und Trennung ist. Im Beispiel präzisieren Gestik und Sprache einander also nicht einfach wechselseitig, wie dies für das Verhältnis von Zeigegesten und sprachlichen Deiktika sonst üblich ist, sondern die Gestik bearbeitet eine zweite Dimension des Raums, die von der ersten unabhängig ist und einen eigenständigen Beitrag zu Ausgestaltung der Szene leistet. Dabei scheint charakteristisch, dass genau diejenigen Raumverhältnisse sprachlich codiert sind, welche für den übergeordneten Darstellungszweck, nämlich die räumliche Verdeutlichung der Konfliktdramaturgie, konstitutiv sind. Den Gesten dagegen kommt ihnen gegenüber nur eine weiter ausgestaltende Funktion zu. Diese Verteilung scheint zu reflektieren, dass (im untersuchten Beispiel) in Bezug auf den imaginären Raum die deskriptive Sprache gegenüber der Gestik die tragfähigere, die Verständigung eindeutiger sichernde Ressource der Referenzkonstitution ist, weshalb sie die Aufgabe übernimmt, die für den Darstellungszweck zentralen räumlichen Gegebenheiten darzustellen. ${ }^{8}$ Eine umgekehrte Aufgabenverteilung zwischen Sprache und Gestik scheint in Bezug auf imaginäre Räume, die nicht graphisch o.ä. repräsentiert sind, schlecht vorstellbar.

$8 \quad$ Wichtig für dieses Argument ist, dass die sprachlichen Ausdrücke nicht nur deiktisch sind, sondern hinreichend deskriptiven, symbolischen Gehalt haben, um einen in sich qualitativ ausdifferenzierten Raum zu konzeptualisieren. 
c) Leibliche Enaktierungen der Bewegung im Raum über die Gesten hinaus: Die Inszenierung des Gehens der Gang mit dem Oberkörper (raumbeanspruchendes Verhalten), der Blick der zweiten Gang zur ersten, die Boxkampfgeste, die ermahnende Zeigefingergeste des Sheriffs/Prinzen sind Enaktierungen der Figuren. Sie eröffnen zusätzlich zu Nähe-Distanz und links-rechts eine weitere Ebene räumlicher Information, nämlich die qualitative Ausdifferenzierung der konkreten leiblichen Inkarnationen sozialer und handlungsbezogener Typik.

d) Räumliche Verhältnisse werden sprachlich nicht nur durch ein auf Raumreferenz spezialisiertes Vokabular verdeutlicht. Soziale Kategorisierungen und Handlungsbeschreibungen haben ebenfalls räumliche Implikationen. Der Raum ist auch ein sozial codierter: Die Beschreibung sozialer Handlungen wie „Da geht 'ne Gang die Straße runter.“, die onomatopoetische Charakterisierung des körperlichen Konflikts boff und das Dazwischengehen des Prinzen/Sheriffs gestalten den imaginären Raum sozial und räumlich aus. Der Mitvollzug dieses sozialräumlichen Szenarios erfordert ein Wissen um sozial-räumliche Handlungsskripts (vgl. Goodwin 2003): Z.B. muss dem Zuhörer klar sein, dass das Gehen einer Gang einen Territorium beanspruchenden gesturalen Habitus impliziert (den das Gehen einer Krankenschwester bspw. nicht implizieren würde), und dass boff einen weiteren Eskalationsschritt zum physischen Konflikt bedeutet, welcher die räumliche Konstellation dahingehend vorantreibt, dass die Gangs nunmehr in unmittelbarem Körperkontakt geraten sind.

Damit die unterschiedlichen multimodalen Verfahren der Raumkonstitution allerdings so zusammenwirken können, dass sich für den Rezipienten ein zunehmend ausgestalteter imaginärer Raum entwickelt, ist zusätzlich erforderlich, dass der Rezipient sie als kohärente Ereignisfolge interpretiert. Diese temporalen Kohärenzverhältnisse werden nämlich nicht explizit, etwa durch Konnektoren und temporale Adverbiale wie und dann, nachdem, als nächstes etc., codiert. Nur unter einer temporalen Kohärenzpräsumption erscheinen die einzelnen Äußerungen, Gestikulationen und Enaktierungen des Dozenten nicht als isolierte Setz-Akte in einem amorphen Raum, sondern als konsistent aufeinander bezogene Ausgestaltungen einer konfrontativen sozialräumlichen Konstellation.

\subsection{Konstitutionsverhältnisse und Überblendungen der Räume}

Mit seinen verschiedenen Beteiligungsrollen, die er durch unterschiedliche sprachliche, stimmliche, gestische und enaktierende Verfahren verdeutlicht, macht der Dozent die unterschiedlichen Räume und die in ihnen geltenden Strukturen klar unterscheidbar (vgl. a. Schmitt/Deppermann 2009). Er ist 
1) als Körper im physikalischen Raum mit den andern Körpern kopräsent und

2) sitzt als Dozent mit den Studierenden im gemeinsamen Interaktionsraum.

3) Durch seine kinesische Aktivität in seinem individuellen Verhaltensraum

4) enaktiert er die Figuren auf der Bühne des imaginären Raums,

5) den er als Erzähler innerhalb des Interaktionsraums konstituiert hat.

Der imaginäre Raum wird also durch zwei gänzlich verschiedene Praktiken, nämlich diegetisches Erzählen und leibliches Enaktieren innerhalb des imaginären Raums als Bühne realisiert. Diese beiden Praktiken erzeugen zwei Sprecherrollen (Erzähler vs. enaktierte Figur), die durch jeweilige Gestik, körperliche Beteiligungsweisen, Stimmprofile und räumliche Origones mit entsprechend unterschiedlichen räumlichen Positionen gekennzeichnet sind.

Obwohl hinsichtlich Beteiligungsstatus des Sprechers und repräsentationalem Bezug der Darstellung die Räume klar zu unterscheiden sind, gibt es im untersuchten Interaktionsausschnitt Momente, in denen sie einander überblenden. Eine solche Überblendung im Sinne eines conceptual blends findet zum einen zwischen Erzählen und Enaktieren statt. ${ }^{9}$ Im boff des Dozenten und in der dazu von ihm eingenommene Boxergeste überblenden sich Erzähler und Figur: Mit der Geste enaktiert er die Kampfgeste der Konfliktparteien, der Ausdruck boff ikonisiert den Lärm des Zusammenpralls - soweit agiert der Dozent auf der Bühne; doch andererseits sagt natürlich keiner der Kämpfer boff, sondern boff fungiert als Beschreibung und ist damit Teil der Narration. Und der Dozent stellt hier kein identifizierbares Individuum, sondern einen generalisierten Kämpfer dar. Eine zweite Überblendung, diesmal zwischen Interaktionsraum und Bühne, entsteht in der Ermahnung des Prinzen. Die heranholende Geste mit dem gekrümmten Zeigefinger und die anschließende mahnende Zeigefingergeste, die der verbalen Ermahnung des Sheriffs/Prinzen vorausgehen, sind zunächst einmal nicht bzw. nicht nur im imaginären Raum vom enaktierten Sheriff/Prinz auf die Streitparteien als intendiertem Referenten gerichtet. Sie werden im Interaktionsraum vom Lehrer genau auf den Drehbuchautor, dem die Belehrung gilt, als Demonstratum gerichtet, der damit im Interaktionsraum als primärer Adressat der Belehrung definiert wird. Solche Überblendungen von Räumen sind lokal scharf begrenzte Phänomene, die eine stabile Struktur von Räumlichkeit voraussetzen, bezogen auf die sie kontrastiv zur Gel-

$9 \quad$ Es handelt sich um conceptual blending im Sinne von Fauconnier/Turner (2002) und Turner (2007). Beim conceptual blending werden Komponenten zweier konzeptueller Domänen miteinander zu einem neuen Konzept verschmolzen. Im Gegensatz zur Metapher handelt es sich nicht um eine Übertragung der Verhältnisse von einer Quelldomäne in eine Zieldomäne, sondern um die Amalgamierung von ausgewählten Aspekten aus zwei verschiedenen Domänen, während andere Aspekte aus den Ursprungsdomänen nicht in den blend eingehen. 
tung kommen können. Im Fall der Ermahnung zeigt sich dabei, dass das semiotische Verhältnis unterschiedlicher Ausdrucksressourcen (hier: Sprechen und Gestik) nicht statisch gesehen werden darf, sondern in Abhängigkeit von den pragmatischen Strukturen der Interaktion (hier: der übergeordneten Funktion der Beispielkonstruktion als Vermittlungsverfahren für einen spezifischen Adressaten) rekonstruiert werden muss.

\section{Raum als Ressource des recipient design}

Dass der Dozent zur Vermittlung des Konzepts dramatischer „Struktur“ ein räumlich strukturiertes, narrativ inszeniertes Beispiel realisiert, ist keineswegs zwangsläufig. Er hätte auch die Möglichkeit gehabt, eine abstrakte Beschreibung zu liefern. Die Wahl einer narrativ-enaktierenden Beispielrealisierung reagiert auf die eingangs rekapitulierte Interaktionsgeschichte der gescheiterten Vermittlungsbemühungen. Sie ist damit recipient designed, d.h., an den spezifischen Rezipienten (den Drehbuchautor) und dessen Reaktionen und sein aus ihnen zu erschließendes Wissen angepasst, während sie zugleich diesen als einen bestimmten Rezipienten mit bestimmten Eigenschaften positioniert. ${ }^{10}$ Recipient design ist ein konversationsanalytisches Konzept, das von Sacks (1992) in den frühen 1970er Jahren entwickelt wurde (vgl. Malone 1997, S. 100-119). Sacks/Schegloff/Jefferson (1974, S. 727) definieren es wie folgt:

By ,recipient design' we refer to a multitude of respects in which the talk by a party in a conversation is constructed or designed in ways which display an orientation and sensitivity to the particular other(s) who are co-participants. In our work, we have found recipient design to operate with regard to word selection, topic selection, admissibility and ordering of sequences, options and obligations for starting and terminating conversations etc., as will be reported in future publications.

Die Beispielkonstitution ist ein auf den Studenten bezogenes, „spätes“ Vermittlungsverfahren, das dem Scheitern der vorangegangenen Versuche, ihn zur Einsicht in die Unzulänglichkeit seines dramaturgischen Strukturbegriffs zu bringen, und seiner uneinsichtigen Reaktion auf diese Versuche Rechnung trägt. Die Raumkonstitution gewinnt in unseren Daten ihre spezifische Funktionalität also als Verfahren des recipient design durch ihren spezifischen Kontrast zu den vorangegangenen, begrifflich und argumentativ-maieutisch ausgerichteten Vermittlungsbemühungen. Raumreferenz wird hier als eine Ressource für andere, nicht der räumlichen Orientierung und Lokalisierung dienende Interaktionszwecke eingesetzt. Die Raumkonstitution ist funktional auf den Interaktionsverlauf der gescheiterten Vermitt-

10 Die narrative Performance wird in Schmitt/Deppermann (2009) ausführlicher in Bezug auf ihr recipient design analysiert. Zum Konzept der (Fremd-)Positionierung siehe Lucius-Hoene/ Deppermann (2002, Kap. 8.2; 2004). 
lung, die vorangehenden Reaktionen des Drehbuchautors und die Interaktionsaufgaben des Pitchings im didaktischen Kontext der Filmhochschule bezogen.

Diese funktionelle Motivation der Raumkonstitution bestimmt jedoch nicht nur ihren interaktiven Zweck. Vielmehr können wir sehen, wie die einzelnen Details der Raumkonstitution genau dieser übergeordneten pragmatischen Zwecksetzung, der sie sich verdankt, angepasst sind. Der Dozent macht nicht einfach ein Beispiel, sondern er führt konkret vor, wie eine Exposition filmisch ausgestaltet wird. Das verlangt eine räumlich-sozial gestaltete Szene mit Akteuren, die durch ihr Handeln gleich zu Beginn die Konfliktstruktur des gesamten Filmes verdeutlichen. Dabei transformiert er die unübersichtliche Situation der Exposition der filmischen Version von „Romeo und Julia“" auf ihre sozial-räumliche Grundstruktur. So erleichtert er die Erkenntnis der dramaturgischen Grundstruktur eines in der Exposition angelegten, das Drama vorantreibenden Konflikts. Ganz im Sinne der leichten Erkenntnis strukturiert der Dozent den imaginären Raum Schritt für Schritt und in klar oppositiver Anlage der Figuren und ihrer Handlungen. Die Art und Weise der sprachlichen Raumkonstitution reflektiert genau, wozu sie produziert wird: Es wird nur genau so viel an sprachlicher Raumreferenz vorgenommen wie zur Darlegung der Konfliktdramaturgie notwendig ist. Alle zusätzlichen Informationen, die z.B. notwendig wären, wenn man eine genaue Skizze zeichnen, die Kontrahenten in geographisch bestimmbaren Bereichen lokalisieren oder ihre Herkunft und ihre räumliche Position nach der Konfliktschlichtung erfahren wollte, unterbleiben. Wir rechnen damit, dass diese Anpassung des Umfangs und der Präzision der Raumkonstitution an den übergeordneten pragmatischen Darstellungszweck nicht nur in unseren Daten zu finden ist, sondern ein generelles Merkmal von Raumreferenz und Raumbeschreibungen ist, das bisher nur weitgehend unbemerkt blieb, da diese Aufgaben stets als informationeller Selbstzweck gegeben waren, nicht aber - wie hier - im Dienste anderer Interaktionsaufgaben standen. Dies ist eine Hypothese, die in weiteren Forschungen zu testen wäre. ${ }^{11}$

Die besondere Eignung von Raumkonstitution als Ressource des recipient design in unserem Falle sind also

- die Möglichkeit, ein Problem erfahrungsnah und kognitiv relativ voraussetzungslos zu veranschaulichen;

- das Potenzial, die dramatische Grundstruktur von Opposition und Konflikt metonymisch zu verdeutlichen;

11 Schon lange bekannt ist allerdings eine verwandte Form des recipient design von Raumbeschreibungen an pragmatische Relevanzen, nämlich die Adaptation der Termini der Raumbeschreibung an das Vorwissen des Kommunikationspartners (vgl. Schegloff 1972; Clark/ Wilkes-Gibbs 1986; Isaacs/Clark 1987). 
- die räumliche Inkarnation sozialer Kodierungen, die nicht ausführlich beschrieben wird, sondern durch die Darstellung der kategoriengebundenen Handlungen der Figuren assoziationsreich (,inference-rich“, Sacks 1992, S. 40) evoziert werden kann.

Der Dozent nutzt also die besonderen performativen, kognitiven und experientiellen Potenziale der Raumkonstitution konsequent für die Verfolgung eines didaktischen Zwecks in der Interaktion mit einem spezifischen Rezipienten in Anpassung an das ihm zugeschriebene Verstehensproblem und die ihm zugeschriebenen Verstehenskapazitäten. Er reagiert damit also genau auf den Rezipienten, als der sich der Drehbuchautor in der zurückliegenden Interaktion selbst positioniert und angeboten hat.

\section{Anhang (analysierter Transkriptausschnitt)}

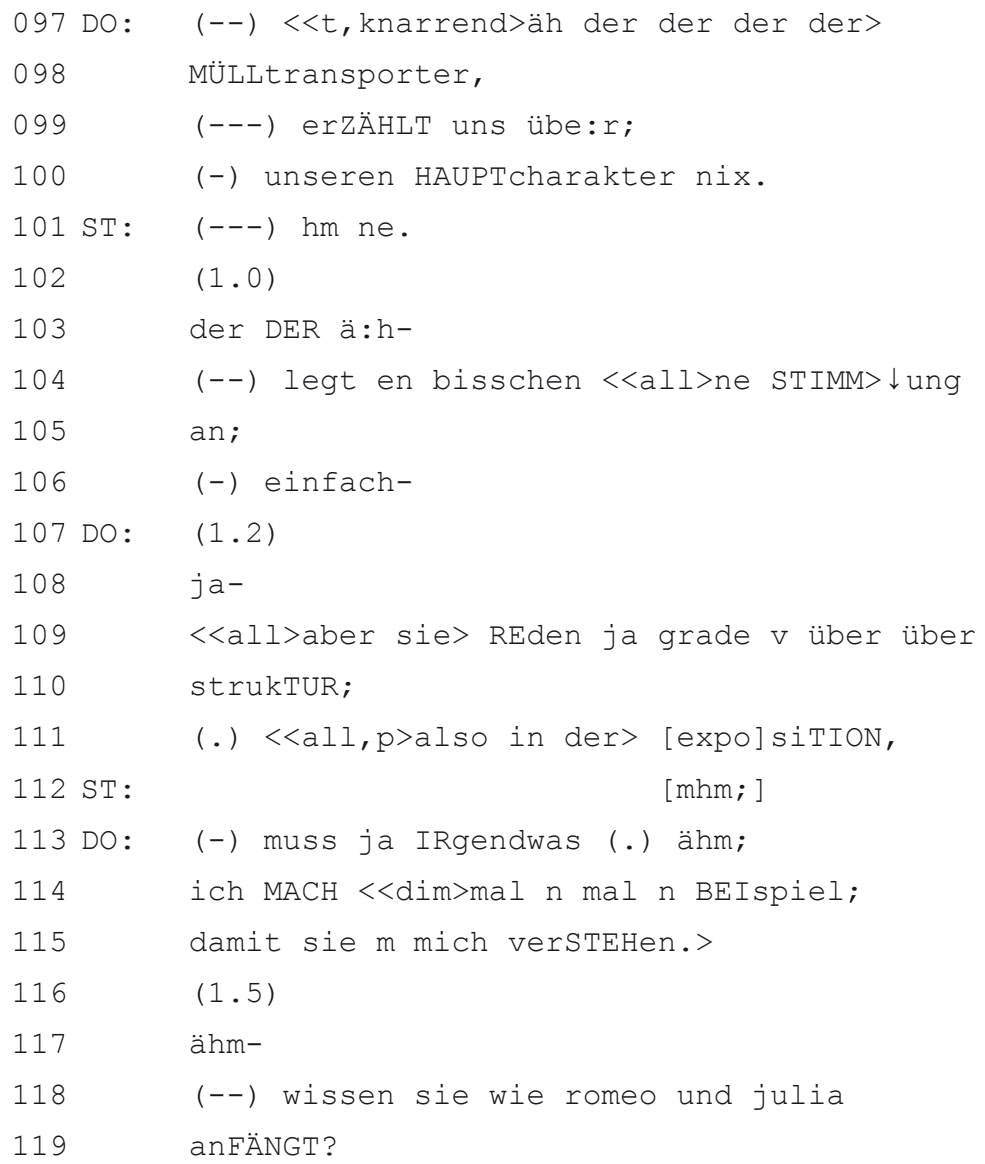


$120 \mathrm{ST}$

$(1.0)$

121

$h m::[: \quad]$

$122 \mathrm{DO}:$

[okay; ]

123

da $<<$ dim $>$ GEHT ne GANG die STRAße runter; $>$

124

(-) $[j a ?]$

$125 \mathrm{ST}:$

['mhm?]

126 DO: $\quad(--)<<t>$ ey-

$127(--)$ was 5 LOS alder;

128 WO is randale; $>$

$129 \quad(-)$ nich?

$130 \mathrm{ST}: \quad(-) \mathrm{mhm}$ ?

131 DO: (--) da kommt ne ANdere gang und sagt $<<$ h $>$ EY MANN DA geNAU (.)

133 auf EUCH ham wir geWARtet; $>$

134

(-) ja?

135

(1.2)

136

boff;

137

(--) die einen sind die CApulets,

138

$(-) \quad<$ dim>und die anderen sind die

139

DINGsens da;>

140

$(--)$ und?

141

$(--)$ wer geht dazWISCHen?

$142 \mathrm{ST}:(--) \mathrm{mh}$;

143 DO: (-) der SHEriff geht dazwischen;

144

der PRINZ geht <<all>dazwischen und sagt; >

145

$<<$ p leute;

146

PASST mal auf;

147

(--) wenn das noch EI:Nmal passiert hier;

148

(-) dann-

149

(1.7)

150

fliecht ihr hier aus der geMEINde;

151

(-) dann werdet ihr Vogelfrei; $>$

$152 \mathrm{ST}:(---)$ ' mhm;

153

(1.2)

$((\ldots))$ 


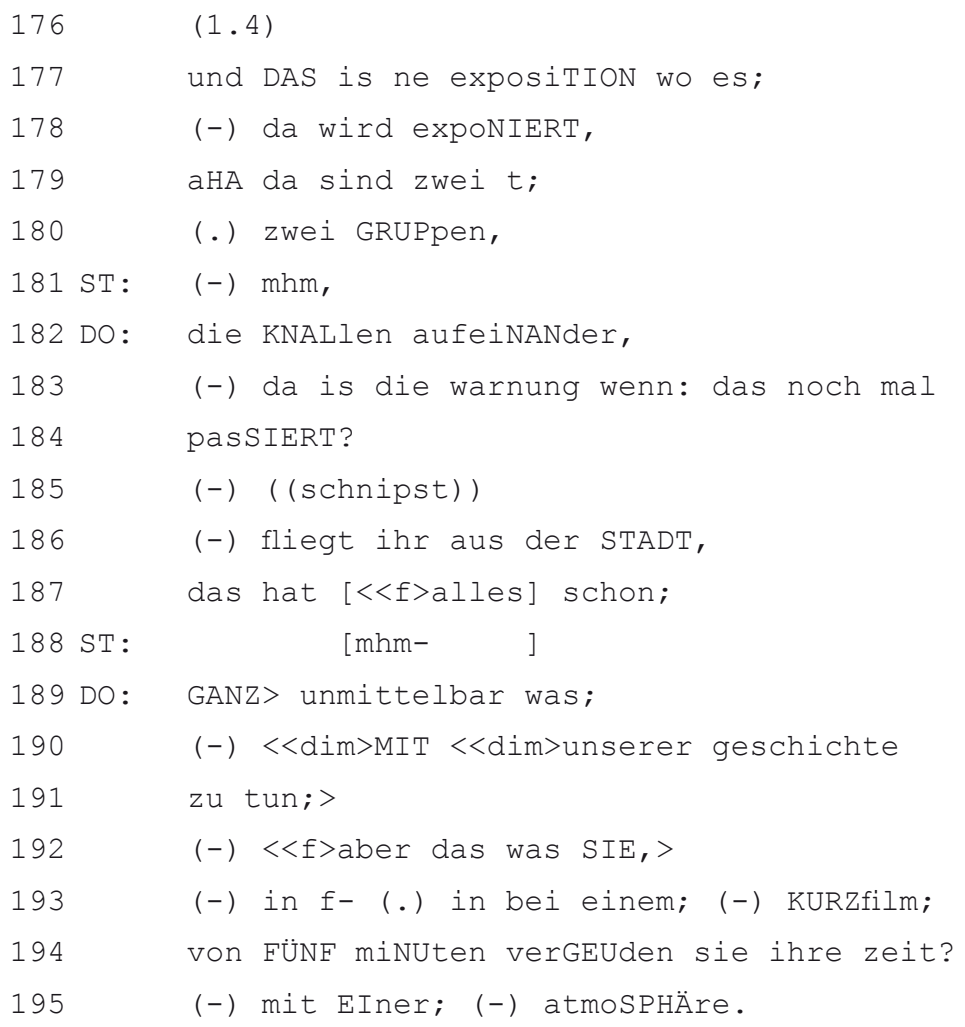

\section{Literatur}

Berthele, Raphael (2007): Ort und Weg. Die sprachliche Raumreferenz in Varietäten des Deutschen. Rätoromanischen und Französischen. Berlin.

Blühdorn, Hardarik (2003): Rauminformation und Demonstrativität. In: Deutsche Sprache 30, S. 252-275.

Bühler, Karl (1934): Sprachtheorie. Jena.

Clark, Herbert H. (1973): Space, time, semantics, and the child. In: Moore, Timothy E. (Hg.): Cognitive development and the acquisition of language. New York, S. 28-64.

Clark, Herbert H./Wilkes-Gibbs, Deanna (1986): Referring as a collaborative process. In: Cognition 22, S. 1-39.

Fauconnier, Gilles/Turner, Mark (2002): The way we think. Conceptual blending and the mind's hidden complexities. New York.

Fillmore, Charles (1997): Lectures on deixis. Stanford, CA.

Fricke, Ellen (2007): Geste, Origo, Raum. Berlin. 
Goodwin, Charles (2003): Pointing as situated practice. In Kita, Sotaro (Hg.): Pointing: Where language, culture and cognition meet. Mahwah, NJ, S. 217-41.

Goffman, Erving (1961): Encounters: Two studies in the sociology of interaction. Indianapolis.

Goffman, Erving (1963): Behaviour in public places. New York.

Goffman, Erving (1981): Footing. In: Goffman, Erving: Forms of talk. Philadelphia, S. 124-159.

Goodwin, Charles/Goodwin, Marjorie Harness (2004): Participation. In: Duranti, Alessandro (Hg.): A compagnion to linguistic anthropology. Oxford, S. 222-244.

Hall, Edward T. (1966): The hidden dimension. Garden City, NY.

Hanke, Mike (1991): maieutike techne. Zum Modell der sokratischen Gesprächstechnik. In: Flader, Dieter (Hg.): Verbale Interaktion. Stuttgart, S. 50-91.

Haspelmath, Martin (1997): From space to time. Temporal adverbials in the world's languages. München.

Hausendorf, Heiko (2003): Deixis and speech situation revisited. The mechanism of perceived perception. In: Lenz, Friedrich (Hg.): Deictic conceptualization of space, time, and person. Amsterdam, S. 249-269.

Hausendorf, Heiko (i.d.Bd.): Interaktion im Raum - Interaktionstheoretische Bemerkungen zu einem vernachlässigten Aspekt von Anwesenheit.

Heidtmann, Daniela (2009): Multimodalität der Kooperation im Lehr-Lern-Diskurs. Wie Ideen für Filme entstehen. (= Studien zur Deutschen Sprache 50). Tübingen.

Herrmann, Theo/Schweizer, Karin (1998): Sprechen über Raum. Bern.

Hopper, Paul J./Traugott, Elizabeth Closs (2003): Grammaticalization. 2. Aufl. Cambridge, UK.

Isaacs, Ellen A./Clark, Herbert H. (1987): References in conversations between experts and novices. In: Journal of Experimental Psychology: General 116, S. 26-37.

Kendon, Adam (1990): Conducting interaction. Cambridge, UK.

Labov, William/Waletzky, Joshua (1973): Erzählanalyse: Mündliche Versionen persönlicher Erfahrung. In: Ihwe, Jens (Hg.): Literaturwissenschaft und Linguistik. Bd. 2. Frankfurt a.M., S. 78-126.

Lakoff, Gordon/Johnson, Mark (1980): Metaphors we live by. Chicago.

Linde, Charlotte/Labov, William (1975): Spatial networks as a site for the study of language and thought. In: Language 51, S. 924-939.

Levinson, Stephen C. (2003): Space in language and cognition. Cambridge, UK.

Lucius-Hoene, Gabriele/Deppermann, Arnulf (2002): Rekonstruktion narrativer Identität. Opladen.

Lucius-Hoene, Gabriele/Deppermann, Arnulf (2004): Narrative Identität und Positionierung. In: Gesprächsforschung 5, S. 166-183. Internet: www.gespraechsforschung -ozs.de/heft2004/ga-lucius.pdf (Stand: 2009). 
Malone, Martin J. (1997): Worlds of talk. The presentation of self in everyday conversation, Cambridge.

McNeill, David (1992): Hand and mind. Chicago.

Mondada, Lorenza (2005): Espace, langage, interaction et cognition: une introduction. In: Intellectica 41-42, S. 7-23.

Mondada, Lorenza (2007): Interaktionsraum und Koordinierung. In: Schmitt (Hg.), S. 55-94.

Sacks, Harvey (1992): Lectures on conversation. Bd. 1. Oxford.

Sacks, Harvey/Schegloff, Emanuel A./Jefferson, Gail (1974): A simplest systematics for the organisation of turn-taking in conversation. In: Language 50, 4, S. 696-735.

Sacks, Harvey/Schegloff, Emanuel A. (2002): Home position. In: Gesture 2, 2, S. 133 146.

Schegloff, Emmanuel A. (1972): Notes on a conversational practice: Formulating place. In: Sudnow, David (Hg.): Studies in social interaction. New York, S. 75-119.

Schegloff, Emmanuel A. (1998): Body torque. In: Social Research 65, 3, S. 535-596.

Schmitt, Reinhold (2003): Inszenieren. Struktur und Funktion eines gesprächsrhetorischen Verfahrens. In: Gesprächsforschung - Onlinezeitschrift zur verbalen Interaktion 4, S. 186-250. Internet: www.gespraechsforschung-ozs.de/heft2003/ga-schmitt. pdf (Stand: Oktober 2009).

Schmitt, Reinhold (Hg.) (2007): Koordination. Analysen zur multimodalen Interaktion. (= Studien zur Deutschen Sprache 38). Tübingen.

Schmitt, Reinhold/Deppermann, Arnulf (2007): Monitoring und Koordination als Voraussetzung der multimodalen Konstitution von Interaktionsräumen. In: Schmitt (Hg.), S. 95-128.

Schmitt, Reinhold/Deppermann, Arnulf (2009): „damit sie mich verstehen“: Genese, Verfahren und recipient design einer narrativen Performance. In: Buss, Mareike/ Habscheid, Stephan/Jautz, Sabine/Liedtke, Frank/Schneider, Jan-Georg (Hg.): Theatralität des sprachlichen Handelns. München, S. 83-112.

Schütz, Alfred (1981): Der sinnhafte Aufbau der sozialen Welt. Eine Einleitung in die verstehende Soziologie. 2. Aufl. Frankfurt a.M.

Scollon, Ron/Wong Scollon, Suzanne (2003): Discourses in place: Language in the material world. London.

Selting, Margret/Auer, Peter/Barden, Birgit/Bergmann, Jörg/Couper-Kuhlen, Elizabeth et al. (1998): Gesprächsanalytisches Transkriptionssystem (GAT). In: Linguistische Berichte 173, S. 91-122.

Sitta, Georg (1991): Deixis am Phantasma. (= Bochumer Beiträge zur Semiotik 31). Bochum.

Tenbrink, Thora (2007): Space, time, and the use of language. (= Cognitive Linguistics Research 36). Berlin.

Turner, Mark (2007): Conceptual integration. In: Geeraerts, Dirk/Cuyckens, Hubert (Hg.): The Oxford handbook of cognitive linguistics. Oxford, S. 377-393. 
